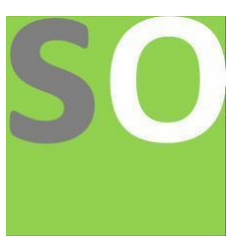

Article title: Trial Interventions in ABCA4- Retinopathy

Authors: Majid Al Breiki[1]

Affiliations: University College London[1]

Orcid ids: 0000-0003-4789-0226[1]

Contact e-mail: al_briki@hotmail.com

License information: This work has been published open access under Creative Commons Attribution License $\mathrm{http}: / / c r e a t i v e c o m m o n s . o r g / l i c e n s e s / b y / 4.0 /$, which permits unrestricted use, distribution, and reproduction in any medium, provided the original work is properly cited. Conditions, terms of use and publishing policy can be found at https://www.scienceopen.com/.

Preprint statement: This article is a preprint and has not been peer-reviewed, under consideration and submitted to ScienceOpen Preprints for open peer review.

DOI: 10.14293/S2199-1006.1.SOR-.PPZONMP.v1

Preprint first posted online: 05 June 2020

Keywords: $A B C A 4$, retinopathy , pathophysiology, $A B C A 4$ transporter physiology 


\title{
Trial Interventions in ABCA4- Retinopathy
}

Majid Al Breiki

\begin{abstract}
$\underline{\text { Abstract }}$
Inherited retinal diseases collectively are one of the leading causes of visual impairment worldwide. ABCA4 retinopathy is the most common inherited retinal disease. In the last few years, there are many advances in the understanding of this disease, which led to many interventional trials with promising results at the moment. This paper is going to present a brief background up to date knowledge in the context of the disease pathophysiology, the main mechanisms involved in this disease and related diseases, and different approaches of the intervention trials. I will be using a literature review to highlight the main structural, physiological, chemical, functional characteristics of ABCA4 transporter physiology. This will be followed by a brief discussion about pathophysiology. Next, analysing, extracting and comparing different interventional options and presenting the current clinical trials for each will be done. This paper expected to discusses and rationally compare between different treatment trials approaches their advantages over others and challenges. A sensible, possible application from current trials from different diseases with similar pathogenesis and possible research opportunities will be discussed.
\end{abstract}




\section{ACKNOWLEDGEMENTS}

I would first like to thank my thesis supervisor: Professor Andrew Webster of the Moorfield's Eye Hospital. He provides me with guidance and support. He was encouraging hroughout, and I want to acknowledge him for his contribution to this work by providing a real-life case of ABCA4 retinopathy.

I would also like to acknowledge the Moorfield eye hospital as this real-life case of ABCA4 retinopathy seen and investigated there. 


\section{Contents}

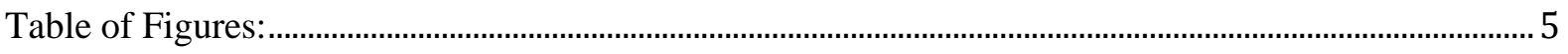

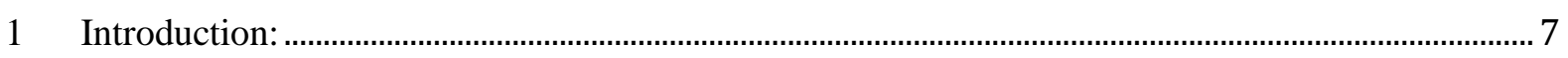

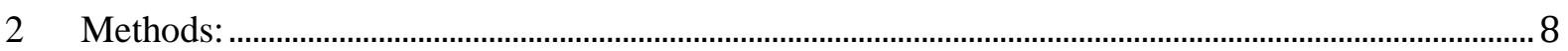

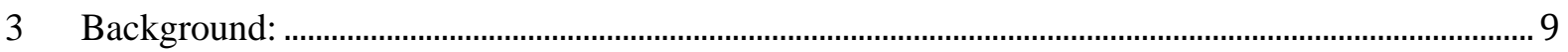

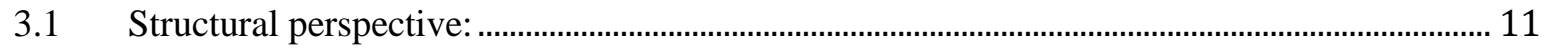

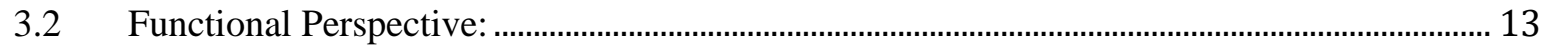

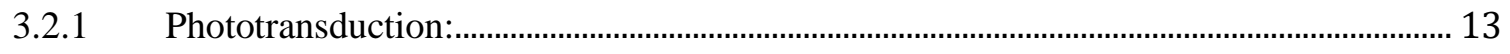

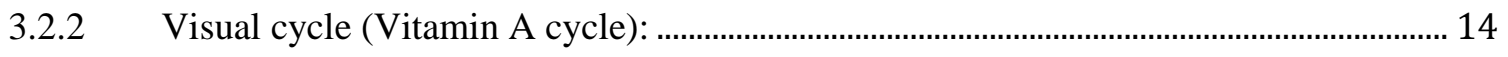

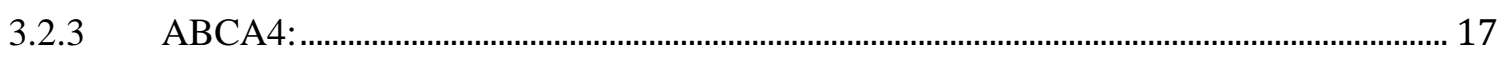

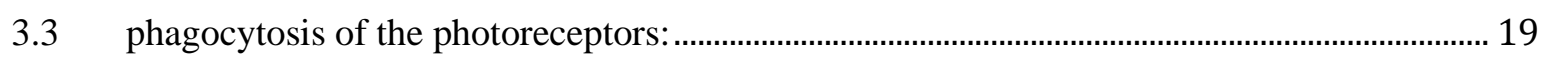

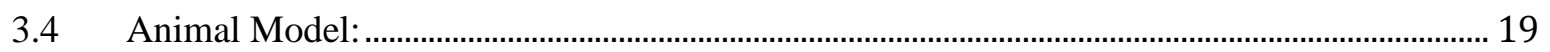

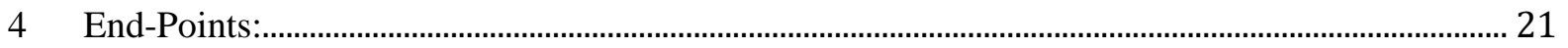

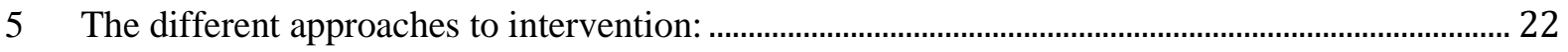

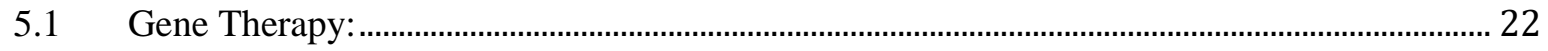

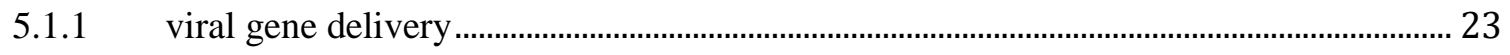

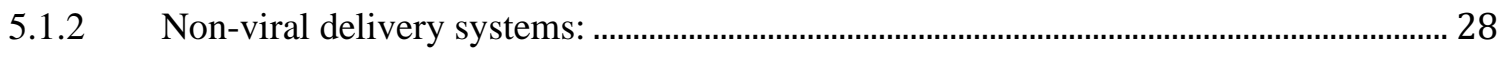

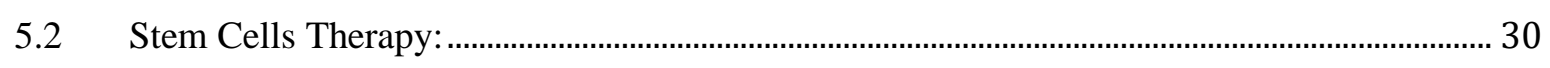

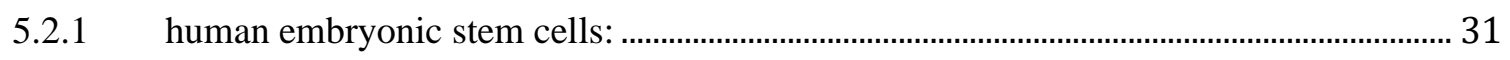

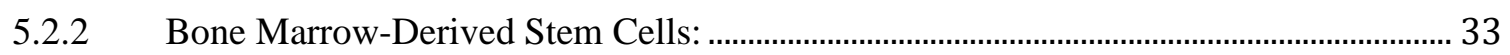

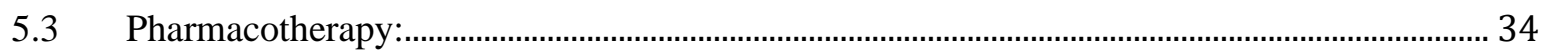

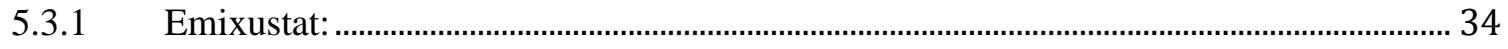

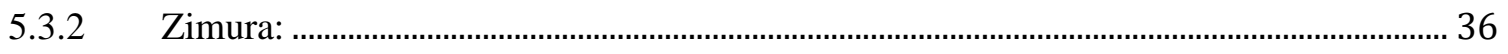

5.3.3 Docosahexaenoic acid: .................................................................................................. 36

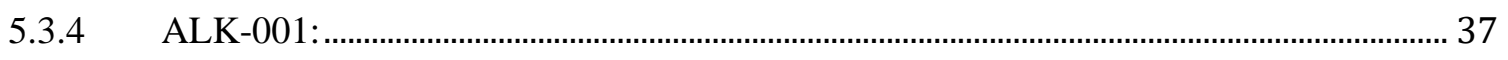

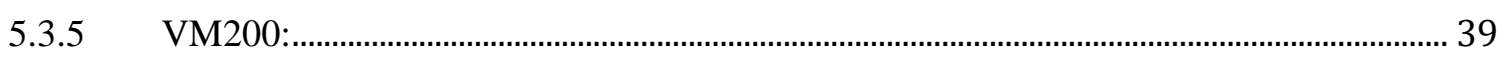

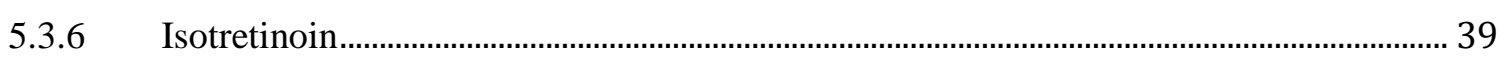

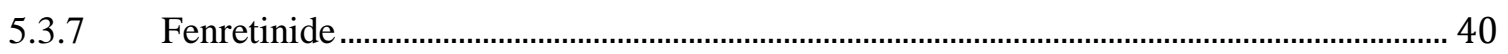

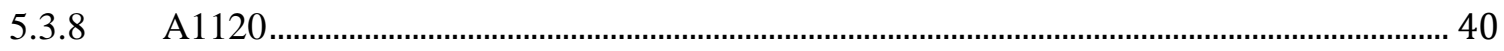

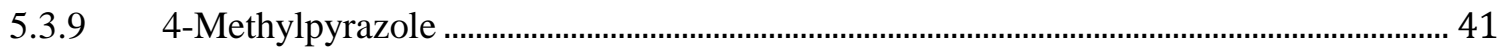

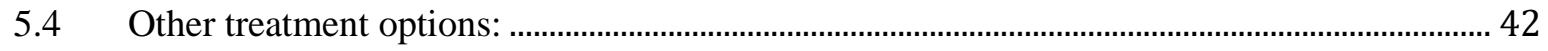

5.4.1 Microcurrent Stimulation Therapy: …………................................................................... 43

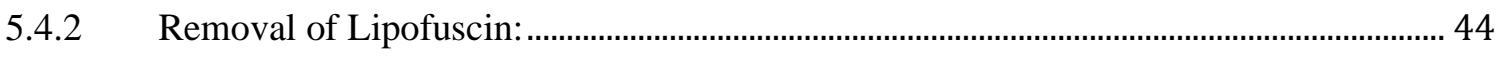

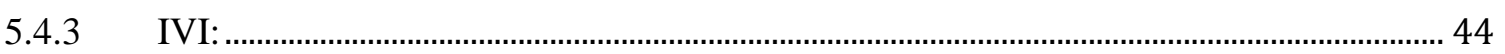




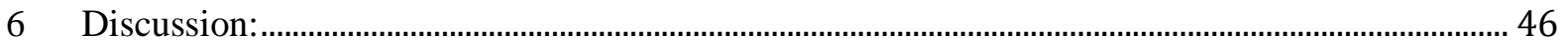

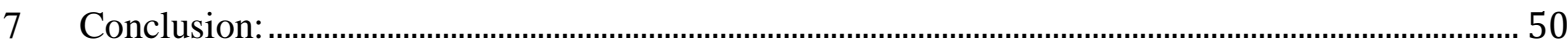




\section{Table of Figures:}

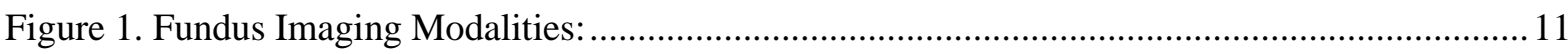

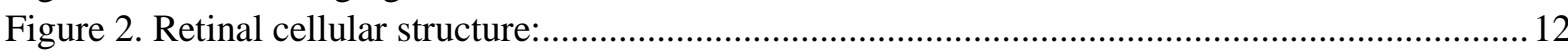

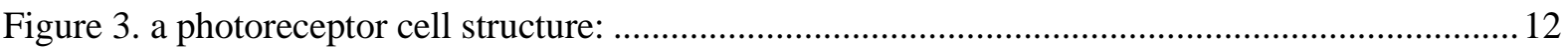

Figure 4. phototransduction cascade:

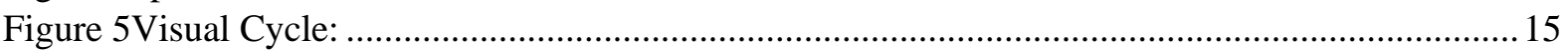

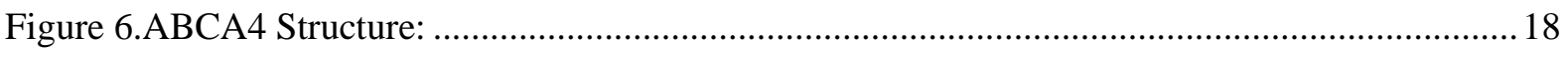

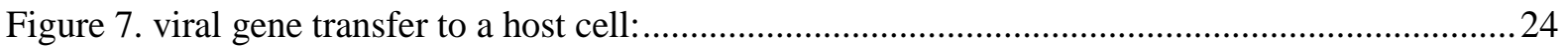

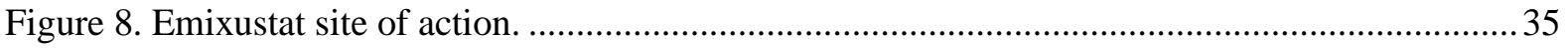

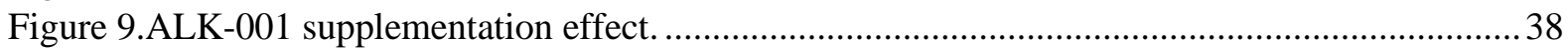

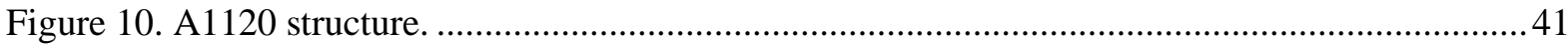




\section{$\underline{\text { Abbreviations list }}$}

\begin{tabular}{|c|c|}
\hline $\mathbf{A} 2 \mathbf{E}$ & N-retinylidene-N-retinyl-ethanolamine \\
\hline A2PE & di-retinoid-pyridinium-phosphatidylethanolamine \\
\hline ABCA4 & ATP-binding cassette transporter, subfamily A, member 4 \\
\hline ABCR & ABCA4 Receptor \\
\hline Ad & Adenovirus \\
\hline $\mathbf{A F}$ & fundus Auto-Fluorescence \\
\hline BCVA & Best Corrected Visual Acuity \\
\hline BMSC & Bone Marrow-Derived Stem Cells \\
\hline ECD & Extracellular Domain \\
\hline ERG & electroretinography \\
\hline hESCs & human embryonic stem cells \\
\hline IRBP & Interstitial Retinal Binding Protein \\
\hline IRD & Inherited Retinal Disorder \\
\hline LCA & Leber Congenital Amaurosis \\
\hline $\mathbf{L V}$ & Lentivirus \\
\hline MII & metarhodopsin II \\
\hline MIM & Mendelian Inheritance in Man \\
\hline NBD & Nucleotide Binding Domains \\
\hline N-ret-PE & N-retinylidene-phosphatidylethanolamine \\
\hline OCT & optical coherence tomography \\
\hline OS & Outer Segment \\
\hline PE & phosphatidylethanolamine \\
\hline PR & Photoreceptor \\
\hline RDH & all-trans-retinol dehydrogenase \\
\hline RPE & Retinal Pigmented Epithelium \\
\hline SD-OCT & spectral-domain optical coherence tomography \\
\hline STGD1 & Stargardt disease \\
\hline TEM & transmission-electron microscope \\
\hline TMD & Transmembrane Domains \\
\hline
\end{tabular}




\section{Introduction:}

In order to appreciate the world, we are living in and interact with it; we need a precise visual system which we can depend-on. Our eyes are very compact, but they serve a vital role in our daily life. There are many diseases which can affect this organ limiting or completely incapacitate it functionally. Inherited Retinal Disorder IRD affects millions of people around the world with no available treatment to any. However, a star lights up the sky as first treatment (LUXTURNA) was approved by FDA in 2017 and EU in 2018 as a one-time treatment for Leber Congenital Amaurosis (LCA) [1]. The most common IRD is Stargardt disease (STGD1) (MIM 248200), which has no available treatment up to date[2-5].

There are many advances in this file, which is supported by related fields improvements, discoveries and innovations. This area is quite active at the moment with many trials being designed, done or analysed and reported. This paper is going to discuss briefly the ABCA4 transporter physiological role in standard photoreceptor cells with great emphasis on the visual cycle, which is essential in understanding the role of each intervention. After that, pathophysiology associated with ABCA4 dysfunction will be discussed. Then, ABCA4 retinopathy will be touched on briefly. Subsequently, different treatment approaches will be explored, and different trials will be included. Finally, a brief rational discussion about these interventions will be done.

By the end of this paper, I hope to answer "What are current intervention trials for ABCA4 retinopathy?". The literature review will be the method which will be used to analyse the question and answer it. 


\section{Methods:}

In this paper, I will be using a literature review to highlight the main structural, physiological, chemical, functional characteristics and genetic aspects about ABCA4 retinopathy. The primary research resources are mainly from peer-reviewed journal articles, academic textbooks, clinical trials records and workshop reports in addition to one case report. No conference papers included. Some broad terms on the subject used to search in the literature. These terms include Stargardt disease, STGD1, ABCA4, ABCR, visual cycle, vitamin A cycle, retinoid cycle and phototransduction cascade. These terms used separately or in conjunction aiming to get relevant articles. Additional more specific terms were used throughout my reading to explore some specific terms in that specific article. The core Academic searches database include PubMed, Google Scholar, Cochrane Library, ClinicalTrials.gov and EU Clinical Trials Register. A specific search is conducted in Genetics Home Reference Library and Online Mendelian Inheritance in Man MIM database. More references were looked for by looking into the reference section in some articles. One article was obtained from the Institute of Ophthalmology library through the librarian help, and appropriate copyright form was filled and submitted. There are 50 references which are published from 1995 to 2019.

A full text of one article was available only in the German language which was then translated to English using https://translate.google.com. There are 16 intervention clinical trials registered at ClinicalTrials.gov website, which all included in this paper in their respective section. The European Union Clinical Trials Register has four clinical trials which all registered in ClinicalTrials.gov registry. 


\section{Background:}

Karl Bruno Stargardt described a new disease entity with some peculiar genetic and neuroepithelial characteristics which are likely to begin by cones involvement initially, followed by the retinal pigment epithelial (RPE) cells and then to the choroid. This description of seven patients in 1909 included their fundus features and familial nature of this disease[6, 7]. After about half a century specifically in 1962, a Schweizer man called Adolf Franceschetti was describing an unusual fundus condition in the German Ophthalmological Society which he named as fundus flavimaculatus. Professor Jules François from Belgium reported 28 cases in 1963 at the French Ophthalmological Society. Eight more cases were also published in the same year by him. A conclusion made by François in 1975 publication stating that "Stargardt disease and funds flavimaculatus were identical disorders with characteristic clinical, electrophysiological, and genetic features" [6].

Stargardt's disease is a juvenile macular degeneration, also known as Stargardt's macular dystrophy, which was first reported in 1909 [6-8]. Stargardt disease type 1 (STGD1; OMIM 248200) is the most common single-gene inherited retinal dystrophy in humans and the most prevalent form of macular degeneration in children[3, 4, 7, 9]. It is an autosomal recessive disease and may lead to legal blindness within the second or third decade of life $[4,7,10,11]$. The estimated prevalence of the disease is 10 to 12.5 per $100,000[4,12]$. This is mostly caused by a mutation in the ATP-binding cassette transporter, subfamily A, member 4 (ABCA4) gene[4, 13, 14]. It is about $250 \mathrm{kDa}$ single-chain transporter which localised at the disk membrane of outer photoreceptor segments.[14]. It is located within chromosome 1p13.5-8 ABCA4, which encodes for the ABCA4 receptor (ABCR) [13]. ABCR acts as a phospholipid flippase which enabling vitamin A derivatives to be transported through the outer segment disc membranes[13].

There are many genetic variabilities and phenotypic diversity within "Stargardt disease" term, which made it difficult to be dealt with. For this reason, in this paper, we are going to specifically discuses ABCA4 retinopathy and current intervention trials going on. ABCA4 retinopathy characterised by a progressive bilateral loss of central vision in the first or second decade of life with poor final visual outcome[7, 15]. Note that, the peripheral visual field remains normal throughout life if not complicated by other pathology.[7]. Clinically, there is RPE atrophy at the macula, orange-yellow flecks at the mid-periphery, progressive atrophy of photoreceptors and RPE cells, and delay in dark adaptation [7, 11, 15]. 
ABCA4 retinopathy is characterised histologically by a massive accumulation of toxic bisretinoids in photoreceptor PR outer segments and Retinal Pigmented Epithelium RPE.[15, 16]. This result in RPE cells death and loss of its role in maintaining of PR and phagocytosis of their ageing outer segments. PR degeneration is secondary to RPE loss.[14]

Choosing the correct investigation modalities is essential to come up with the right differential diagnosis. Basic ophthalmic evaluation is a must for all retina clinic patients include: Best Corrected Visual Acuity BCVA, dilated fundus exam, colour fundus photography and Optical Coherence Tomography OCT nowadays becomes one of the routine investigations as well. A more specific investigations may be needed, and their results should be presented in the context of the patient complaint and clinical findings. Fundus Auto-Fluorescence AF is a non-invasive imaging technology that often allows abnormalities to stand out when other modalities break, as demonstrated by the real-life case of 29 years old patient Figure 1 [17]. $\mathrm{AF}$ is used as such to diagnose and monitor disease development in STGD1, which show an increase in the level of autofluorescence in early stages [13, 17]. Visualization of intraretinal architectural morphology has been accomplished using Spectral-Domain OCT (SD-OCT), which was earlier only achieved with histopathology and can act as clinical outcome measures [16]. SD-OCT can offer qualitative and quantitative valuable data about the disease. These data, such as total macular volume (TMV) and ellipsoid zone (EZ) measurements can serve as clinical trials endpoints as discussed in section $4[4,16]$. This was taken to the next level by introducing artificial intelligence (AI) tools, which show $100 \%$ accuracy in predicting the diagnosis of ABCA4 from SD-OCT images [18]. Electrophysiology is essential in disease monitoring tool which can and is being used as a trial endpoint measurement. Microarray and next-generation sequencing are the most common tests used to confirm the disease genetically, and it played a very fundamental rule in the inclusion and exclusion criteria for many trials discussed later. 


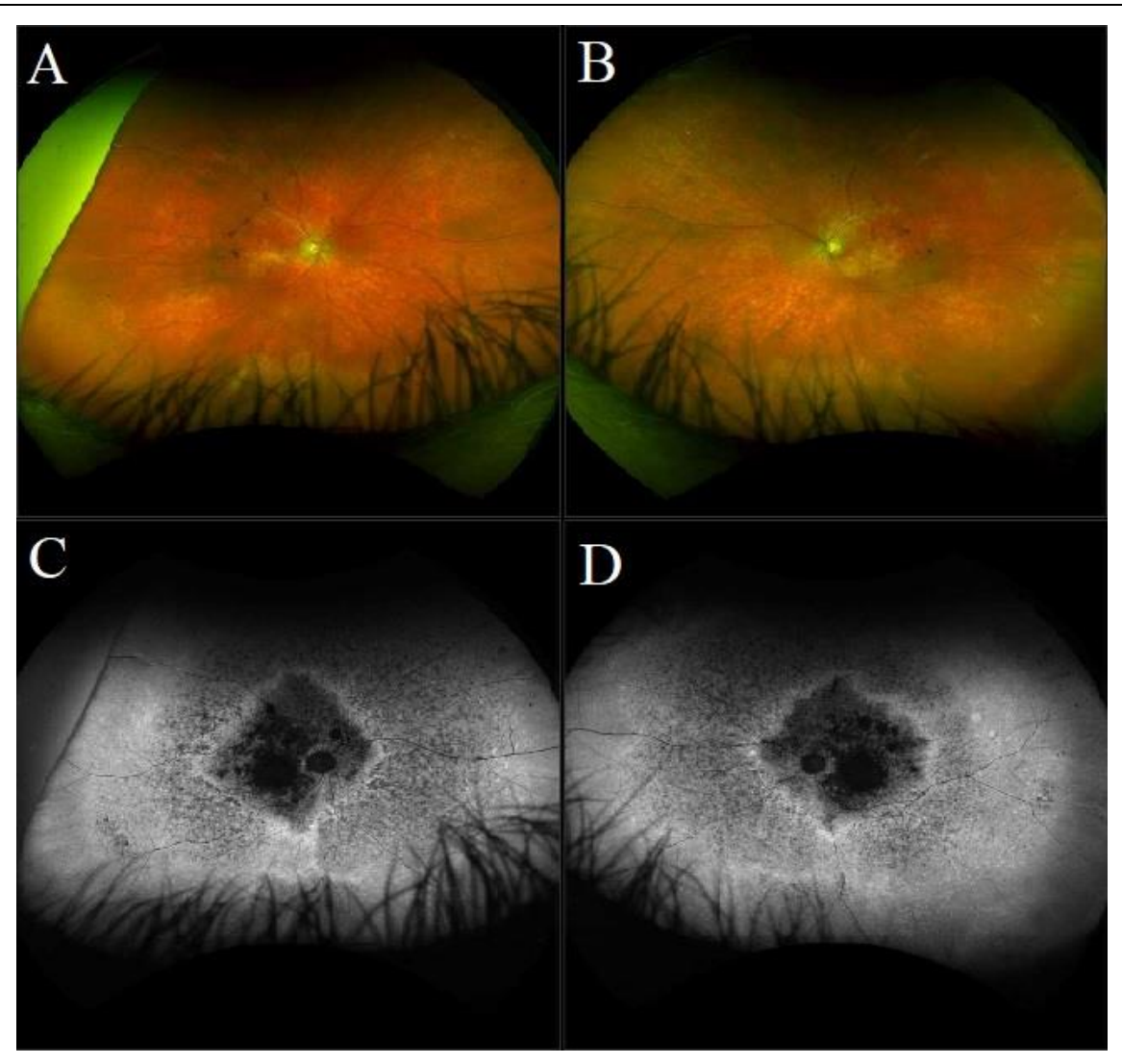

Figure 1. Fundus Imaging Modalities:

This is a 29 years old patient with p.I1100N mutation of the ABCA4 gene. A and B show both eyes wide filed coloured fundus photograph. $\mathrm{C}$ and $\mathrm{D}$ are $\mathrm{AF}$ images for the same patient with a clear demarcation of the lesion. Images provided by Prof Webster from Moorfield Eye Hospital.

\subsection{Structural perspective:}

Rods and Cones are the main two types of photosensitive cells in the retina, which are different anatomically and histologically. Cones are more abundant in the central retina (macula) whereas peripheral retina is rich in rods. Cones are tightly packed with very few rods in the macula [19]. Both types of PR cells can be divided into the inner segment, which composed of the cell bodies and the Outer Segment OS, which is packed with photosensitive membranous discs [19]. These two segments are connected by a non-motile cilium[20]. Rods and cones are supported by RPE, which is a monolayer of hexagonal cells located between the outer segment and choroid. The RPE plays a significant role in providing nutrients for PR cells, regeneration of visual pigment, ingestion and degradation of the OS [11]. Figure 2 shows the microscopic cross-section of the individual retinal layers and the cartoon representation of 
these layers. Figure 3 represents the rod photoreceptor as an example of the photoreceptor cell and the retinal pigment epithelial (RPE) cell.
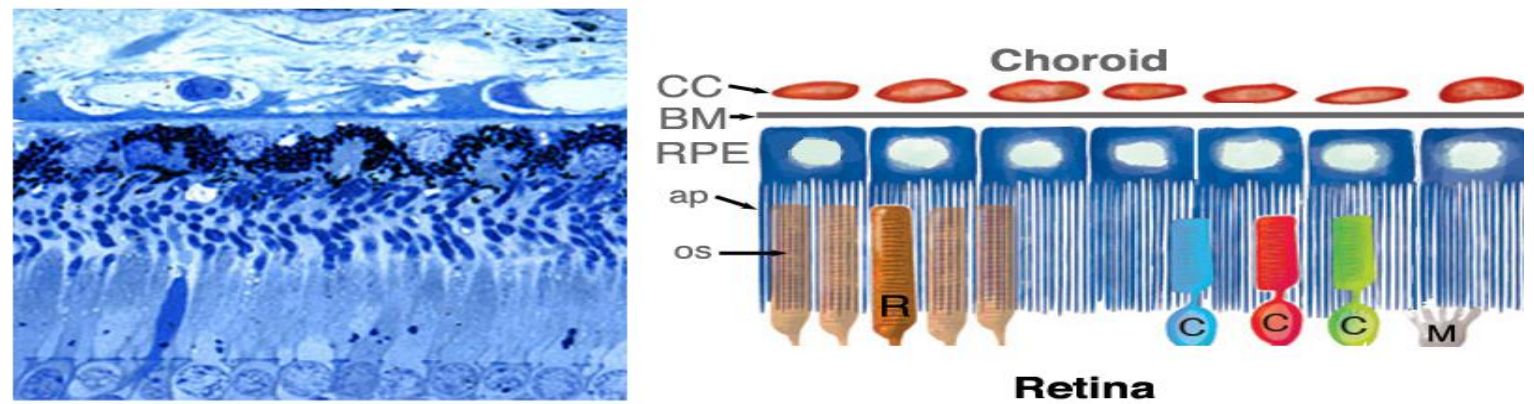

Figure 2. Retinal cellular structure:

This figure is adopted from Kolb et al. [19]. LEFT: a microscopic slid of the retina (LEFT ) and corresponding schematic drawing of these layers (RIGH). CC, Choriocapillaris; BM, Bruch's membrane; RPE. retinal pigment epithelium; ap, apical processes; os, outer segments; C, cones; R, rods, $\mathrm{M}$, muller

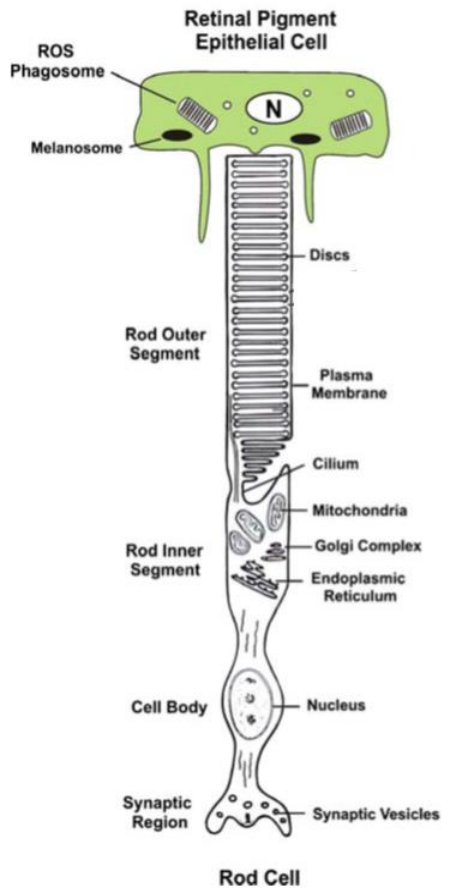

Figure 3. a photoreceptor cell structure:

Diagrammatic representation of rod photoreceptor and retinal pigment epithelial (RPE) cell. RPE contains photoreceptor segment for phagocytosis and renewal. N; nucleus. Adopted from Molday et al. paper [11]. 


\subsection{Functional Perspective:}

There is a close functional interaction between RPE and PRs, which is crucial for a successful visual function. This interaction starts from the embryonic development, which is resembled by the differentiation of these tissues depends on each other [19]. This is continued by providing nutrients, vitamin A supply, waste removal and outer segment renewal are some of these interactions [19]. Besides the structural support provided by RPE, it also for the bloodretinal barrier. Some studies confirmed that mutations in genes which are expressed either of them ultimately affects the other [19].

In this section, we are going to discuss some critical element in understanding the normal physiological functions. Phototransduction cascade is the mechanism in which the eye transfers a perceived light into a neural signal. This is carried out in the outer photoreceptor segment. To keep this cascade running it requires photopigment called 11-cis-retinal which is stored and re-isomerised in RPE by the visual cycle. Visual cycle and the ABCA4 role will be explored in the following pages.

\subsubsection{Phototransduction:}

Vision starts by absorption of a single photon by a light-sensitive molecule called rhodopsin which is formed by opsin and the chromophore molecules. 11-cis-retinal is the chromophore for most opsins [20]. Because of photoisomerisation, 11-cis-retinal will change its conformation into all-trans-retinal and rhodopsin will convert to meta-rhodopsin (MII), and they will dissociate from each other [20]. MII will undergo additional reaction steps before it binds to a new 11-cis-retinal molecule, and it can be activated again by a photon [20]. The previous reaction will lead to activation of intermediate molecules, which lead ultimately to hyperpolarisation and a generation of a neuronal signal from a photochemical reaction[20, 21]. This cascade is summarised in Figure 4.Therefore; a continues supply of 11-cis-retinal need to be available for all visual needs and proper visual function[21]. 


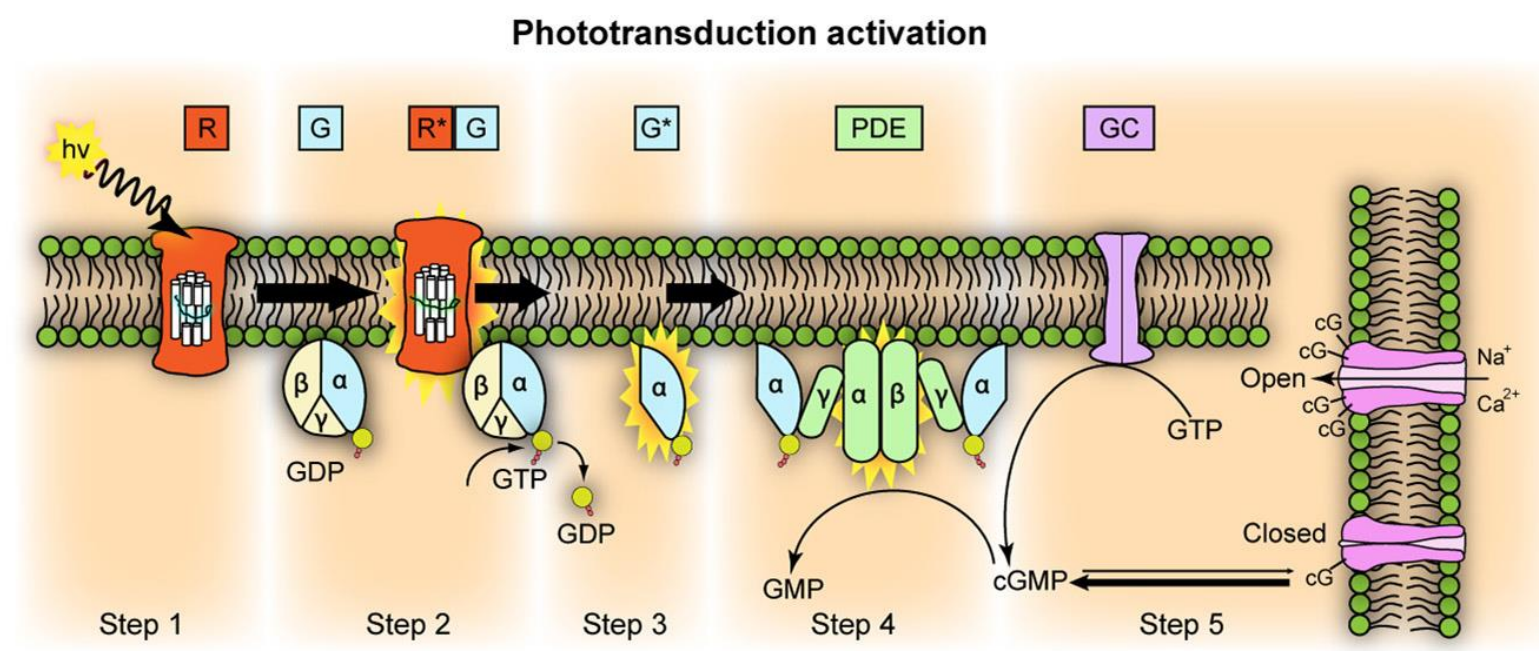

Figure 4. phototransduction cascade:

This figure illustrates the various steps involved in the phototransduction cascade. It starts by a photon activate rhodopsin and going through many intermediate steps ending by the closure of gated channels, preventing further influx of $\mathrm{Na}+$ and $\mathrm{Ca} 2+$ cations. It is adapted from Grossniklaus et al. [21].

\subsubsection{Visual cycle (Vitamin A cycle):}

Healthy photoreceptor and RPE cells are required for the visual cycle to take place [10]. All-trans-retinal supply is very crucial for a good functional PR [20]. Visual cycle is very sophisticated processes and reactions which are tightly controlled by enzymes that are encoded on the genome. This genetic information recessively inherited. The visual cycle started in the discs of the OS completed in RPE and supplied back to PR [19]. Müller glia cells thought to play a role in alternative retinoid recycle for Cone PRs [19, 22]. The visual cycle will be broken down into simple steps. Note that the first step is taking place in the discs of the OS with ABCA4 transport all-trans-retinal to the subretinal space [19]. Figure 5 illustrates the different steps in the visual cycle. Here we will go through the main steps in this cascade: 


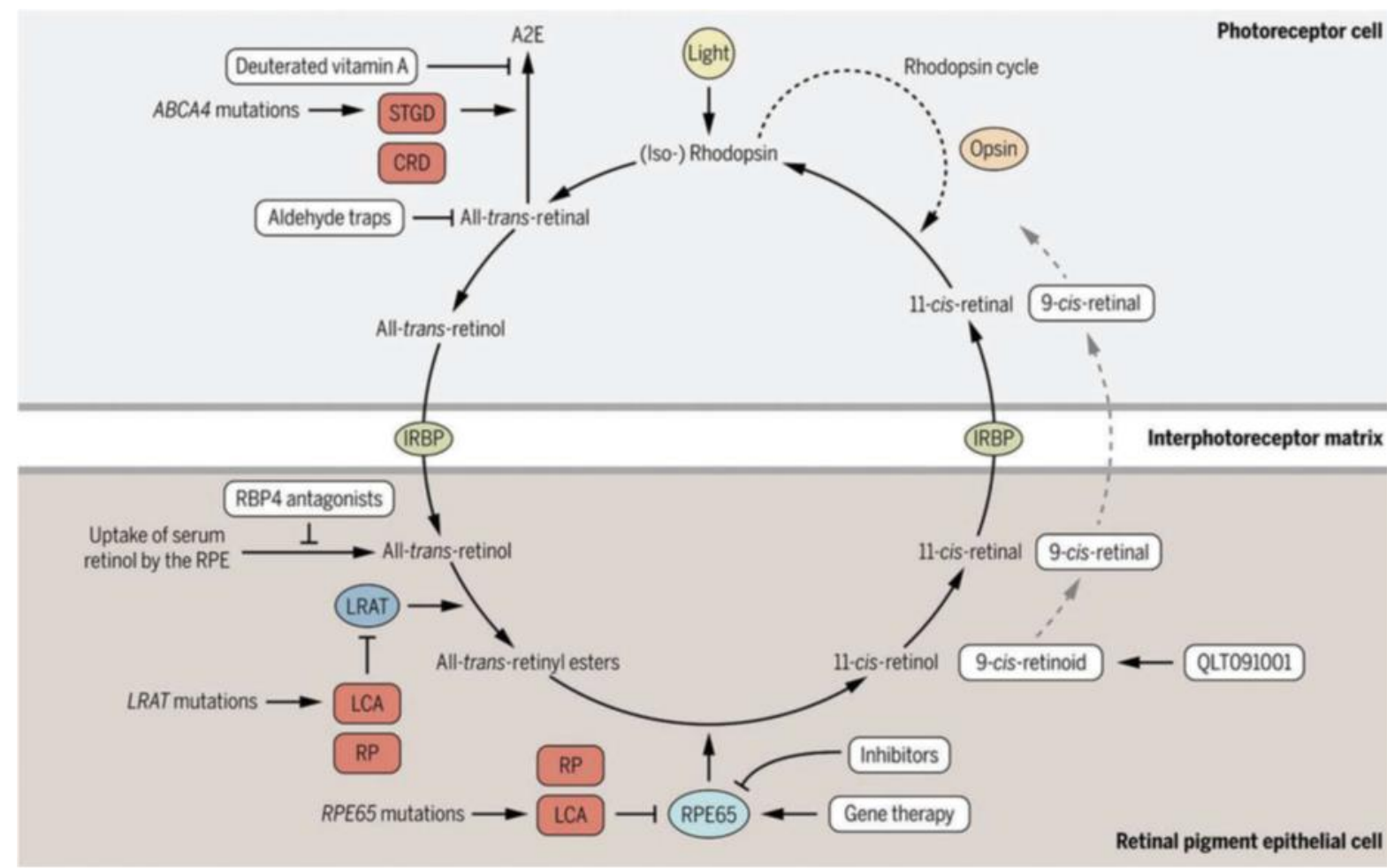

Figure 5Visual Cycle:

This diagram which is adopted from Hussain et al. showing various steps of the visual cycle, which main aim is to convert all-trans-retinal back to 11-cis-retinal and keep phototransduction cascade running [22].

Step 1: All-trans-retinal moved from the disc to cytoplasm:

After MII inactivation, all-trans-retinal will quickly diffuse into the phospholipid bilayer of the disc membrane[14, 20]. Retinal can effortlessly condense with phosphatidylethanolamine (PE) to form a Schiff base which is available in the disc to form N-retinylidenephosphatidylethanolamine (N-ret-PE) [10, 19]. This reversible process can delay all-transretinal clearance from the disc interior to the cytoplasm. ABCA4 role is to actively transfer alltrans-retinal from the intradiscal space to the cytosolic space of the rod OS [19]. Any defect in this transported will result in ABCA4 retinopathy, which is characterised by the accumulation of lipofuscin and reduced supply of 11-cis-retinal. 
Step2: All-trans-retinal to all-trans-retinol:

All-trans-retinal reduction to all-trans-retinol made by a membrane-bound protein called all-trans-retinol dehydrogenase (RDHs) using NADPH as a cofactor [14, 19, 20]. All-transretinol is delivered to the subretinal space/ interphotoreceptor matrix, where it is loaded to a carrier protein Interstitial Retinal Binding Protein IRBP [19, 20].

Step 3: Transfer of all-trans-ROL from subretinal space to RPE Cell:

After the formation of the all-trans-retinol molecule, it is rapidly released by PR cells to the subretinal space. IRBP can bind to both 11-cis-retinol and all-trans-retinal with much higher affinity to the latter one $[19,20]$. There are many mechanisms that facilitate the uptake of alltrans-retinal by RPE cells [20].

Step 4: all-trans-retinol to all-trans-retinyl-esters:

all-trans-retinol is bound to cellular retinol-binding protein CRBP, which act as a substrate for lecithin-retinol-transferase LRAT to transfer an acyl group to all-trans-retinol [19]. This reaction will form an all-trans-retinyl-esters, which is a stable storage form of retinoid [20].

Step 5: all-trans-retinyl-esters to 11-cis-retinol:

Re-isomerization of the 11-cis-retinyl-esters into 11-cis-retinol by RPE protein with $65 \mathrm{kDa}$ (RPE65) is stimulated by binding of 11-cis-retinol to (CRALBP) [19]. RPE65 is inhibited by its product, which is 11-cis-retinol [20]. A newly synthesised 11-cis-retinol molecule can be potentially directed into one of two routes; oxidation to 11-cis-retinal and to be used as a visual chromophore, or it can be stored in 11-cis-retinyl-ester form after esterification [20].

Step 6: 11-cis-retinol to 11-cis-retinal:

By this stage, 11-cis-retinol dehydrogenase type 5 RDH5 catalyse this oxidation reaction to produce 11-cis-retinal in RPE cell [19]. RDH11 is another short-chain dehydrogenase reductase family member in RPE cells that perform the same oxidation reaction. RDH11 may work in harmony with RDH5 to generate 11-cis-retinal [20]. 
Step 7: Back to Photoreceptors:

11-cis-retinal immediately transferred to CRALBP [19]. This step is a regulatory step which keeps an equilibrium as we discussed above in step 5. The exact mechanism of which 11-cis-retinal molecule is transferred from RPE to photoreceptors is not very clear[19, 20].

There are numerous additional details in the visual cycle which regulate the availability of different chromophore molecule forms which could not be covered in this section. It is worth noting that a big part of this knowledge comes from the study of the ABC4 knockout mice studies.

\subsubsection{ABCA4:}

Visual cycle is an enzyme-catalysed reaction that keeps a consistent supply of 11-transretinal for phototransduction cascade. In the first step of the visual cycle (section0), we mention that ABCA4 plays a vital role by transporting all-trans-retinal out of the discs to other enzymes of the visual cycle [10]. ABCA4 was recognised in 1997 as the malfunctioning gene in the Stargardt disease [14, 23].

Previously identified as the PR rim protein (RmP), the ATP-Binding Cassette (ABC) transporter gene, ABCA4 (ABCR), is located at the OS of the PR disc margins $[14,24]$. The human ABCA subfamily includes 12 energy-dependent transporters organised in a 17q24 chromosome cluster $[15,25]$. The most widely studied members of this subfamily are ABCA1 and ABCA4 genes [25]. The ABCA1 protein is associated with an autosomal recessive cholesterol transport disorder and HDL biosynthesis called Tangier disease $[11,25]$. The ABCA4 protein shifts vitamin A derivatives out of the OS, and therefore, it is vital in the visual cycle [25]. The functional protein typically has two halves each contain: one Nucleotide Binding Domains NBD is located on the cytoplasm side, around 6 Transmembrane Domains TMDs, and one extracellular glycosylated Extracellular Domain ECD, as shown in Figure $6[11,14,25]$.

Affected people have reduced visual acuity, impaired colour vision, decreased cone electroretinograms (ERGs), and progressive bilateral atrophy of cone-rich fovea and intrinsic 
retinal pigment epithelium (RPE), It was, therefore, an unexpected consequence that several teams discovered ABCR in rod, but not cone, photoreceptors [26] [15]. Initially, cone loss function was believed to be a secondary result of rod-induced dysfunction of RPE cells [26]. It is stated in the 2002 document that the ABCA4 gene is expressed solely in photoreceptors [25]. It was revealed in a 2017 paper that the gene ABCA4 specifically localises the photoreceptors of rod and cone [9]. A year later, ABCA4 gene was discovered to be expressed not only in the outer segments of the photoreceptor but also in the RPE cells [22]. Surprisingly, an earlier (2010) article revealed several trials identified lower concentrations of ABCA4 expression in the brain tissue in addition to photoreceptors [14].

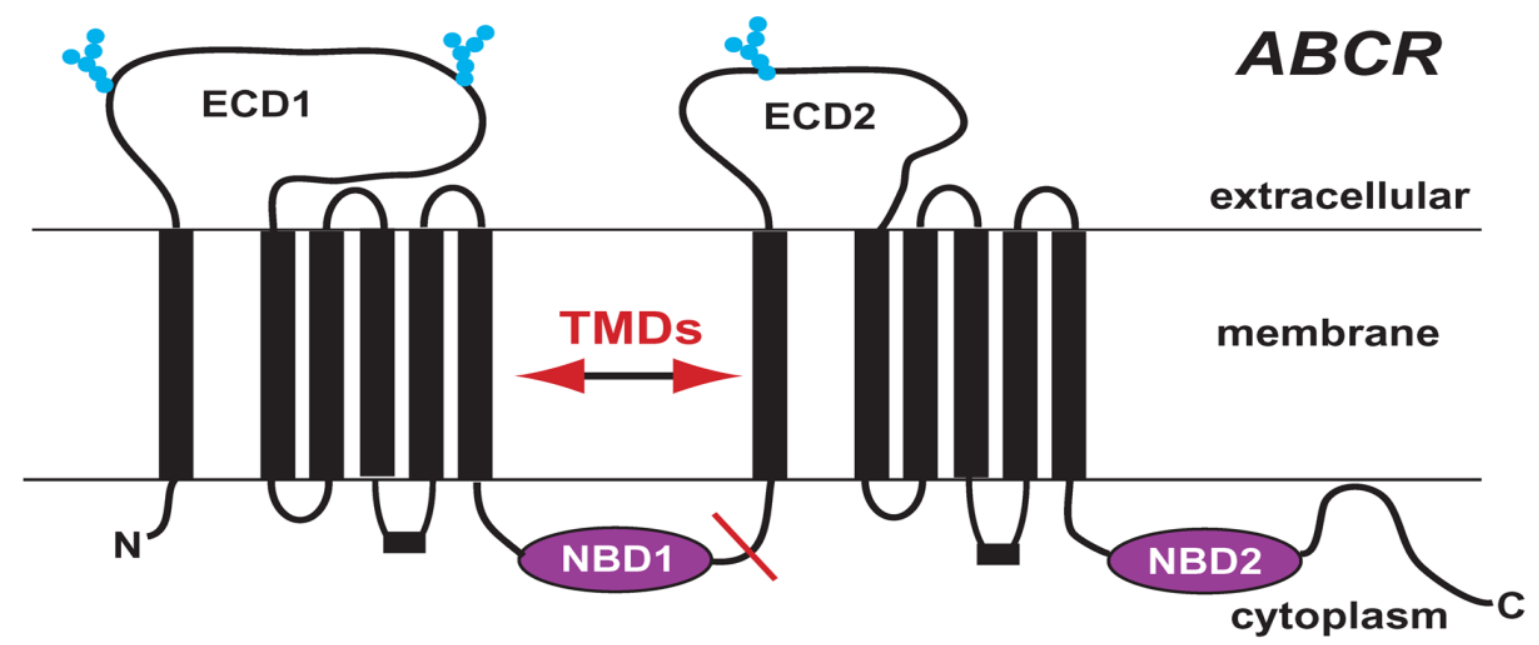

Figure 6.ABCA4 Structure:

This is a schematic structure of ABC transporter showing its $12 \mathrm{TM}$ domains located within the membrane bilayers, adopted from Sullivan et al. [24].

ABCR undergoes a series of conformational changes when ATP binds to the NBDs, bringing the NBDs together and rotating and tilting the TMDs, thereby modulating ligand 
binding to the gated channel [24]. ABCA4 utilises an energy-dependent mechanism to facilitate the removal of all-trans-retinal from the OS by binding and hydrolysis of ATP after their conjugation to PE $[10,24]$. ABCR functions as phospholipid flippase in cone and rod outer segments, allowing the shipping out of vitamin A derivatives through the disc membrane [13]. ABCA4 could thus play its part in shifting N-retinyl-PE to the cytoplasmic side of the disc membrane, allowing all-trans-retinal to go back into the visual cycle [14]. By stopping the accumulation of N-retinylidene-PE inside the disc, ABCA4 would also decrease the response of N-retinylidene-PE to the second molecule of all-trans-retinal, leading to the creation of diretinoid-pyridinium-phosphatidylethanolamine (A2PE) [14].

\section{3 phagocytosis of the photoreceptors:}

The OS is of the light-sensitive portion, which is continually being destroyed due to photooxidative damage $[14,19]$. To preserve sight, OS is continuously renewed by shedding the damaged tips, which are phagocytosed by RPE [19]. This is a diurnally controlled cycle that takes place in the morning and is activated by light [19]. This cycle is controlled not only by the circadian rhythm but also by the interaction between the RPE and the PRs [19]. In RPE, A2PE is hydrolysed to generate N-retinylidene-N-retinyl-ethanolamine A2E as a major

lipofuscin component which cannot be further metabolised [14, 24]. As a result, people with impaired function of ABCA4 gradually accumulate large amounts of A2E in the RPE [14].

Lipofuscin accumulation in RPE is a feature of significant degenerative eye diseases like Stargardt's disease [20, 27]. A2E can have several adverse impacts on RPE cells, including generating reactive oxygen species, impairing lysosomal degrading processes, and interrupting the function of biological membranes In higher concentrations [14].

\subsection{Animal Model:}

It is essential to investigate different therapy approaches on appropriate animal models of disease in preparing for potential clinical trials in Stargardt disease. In 1999, Weng and partners revealed their results about a Stargardt disease knockout mouse model [6]. In the retinal 
pigment epithelium of these mice, they presented a significant deposition of A2E, which is a significant component of lipofuscin [6]. Their research made it possible to determine the function of the Flippase ABCA4 protein mentioned in Section 3.2.3. It was noticeable that Stargardt's knockout mouse model exhibited delayed dark adaptation corresponding to that found in human disease [6]. This research further explained the probable pathogenetic alterations that happen in an animal model of Stargardt which occur in human Stargardt disease as well. A better understanding of disease mechanisms increases the possibility that more effective treatment strategies for specific retinal diseases can be developed and carried out. These conclusions demonstrate the significance of setting up animal models for human eye disease to provide stronger evidence-based counselling to patients regarding their disease management options [6]. 


\section{End-Points:}

The broad combination of variants of ABCA4 and modification factors is virtually unique [2]. This reflects the vast phenotypic heterogeneity, including variable levels of functional and structural improvement, effectively restricting the ability of clinical trials to evaluate the efficacy of novel therapies [2]. Currently, clinical trials usually use functional endpoints to monitor therapy efficacy. The best-corrected visual acuity (BCVA) is one of the most common endpoints widely used [2]. The main disadvantage of BCVA, however, is its extremely variable rate of deterioration in retinal dystrophy patients [2]. Furthermore, visual acuity reduction after an extended duration of pathophysiological change may be a lagging indicator [2].

The primary endpoint of some studies mentioned earlier is the mean rate of change of the area of EZ defects as measured by SD-OCT [28]. Total macular volume (TMV) also suggested by other authors. Another study which we are going to cover in the next section is including BCVA as an endpoint [22]. There are many other endpoints options which used alone or with combination with other options which include: perimetry (static/kinetic), microperimetry, OCT, AF, lesion size, and focal ERG [2]. Retinal degeneration structural measurements may be a more sensitive and reliable clinical endpoint composition [2]. 


\section{The different approaches to intervention:}

At the moment, there is no approved treatment to prevent, delay or give back vision loss for $\mathrm{ABCA} 4$ retinopathy[9]. Using a low-vision aid is used to make the most of their peripheral and paracentral vision. Photoprotection is suggested, but it has its own impracticality [10]. This approach is only to delay the progression of the disease[9]. Currently, there are many ongoing types of research and clinical trials to explore the potential of various new therapeutic possibilities which may successfully bring the scientific and medical society together in preventing vision loss in ABCA4 retinopathy soon [9]. Many trials are under investigation which all share a common goal, which is restricting all-trans-retinal and consequently, the production of $\mathrm{A} 2 \mathrm{E}$ [10]. These include restricting all-trans-retinal formation by limiting light exposure and decreasing intake of vitamin A, improving excretion of vitamin $\mathrm{A}$, and using inhibitors to avoid transportation of vitamin A to the eye. [10].

There are three main approaches to deal with visual cycle gene mutation all aiming to treat the disease successfully:

- Replacing the defective gene.

- Pharmacologically replacing the missing chromophore

- Slowing down chromophore synthesis.

Each approach of these can be tackled in many ways, which all have a final mutual aim[20]. In this section, different therapeutic options will be studied starting by gene therapy, stem cells therapy, pharmacotherapy, any others.

\subsection{Gene Therapy:}

Gene therapy is a promising new approach to treat human diseases. It gains a public interest after FDA approval of the first gene therapy for RPE65 gen in December 2017[1]. The central concept of gene therapy is to introduce a functional copy of the gene instead of a non-functional one [29]. In ABCA4 retinopathy, the goal of gene therapy is to supplement a functional ABCA4 gene to the retina, which may restore the transporter protein functionality [9].

There are several animal models for retinal degeneration with successful gene therapy trials. Gene replacement therapy is an attractive therapeutic option for ABCA4-associated diseases. This approach is a possible "cure" for ABCA4 retinopathy for two reasons [23]: 
- ABCA4 retinopathy is a recessive disease which means replacing one copy of the gen can fully restore visual function.

- There is a decent window for therapeutic intervention in patients with peripheral retinal involvement.

A functional gene needs to be produced, or a non-functional gene needs to be edited or deleted, and a new functional segment needs to be inserted to be done in order to restore the function. For this reason, we need to have a useful gene-editing tool such as CRISPR/CAS tool and an excellent delivery system to the intended site [30]. There are two main ways to deliver the gene by using a viral delivery system or a non-viral delivery system. Each one of these is going to be discussed in this section. Still, all share some regulatory issues which are evolving to cope with the new technologies.

\subsection{1 viral gene delivery}

Having a bioengineered gene sequence which is potentially functioning is not enough to treat a disease. This sequence needs to be delivered to the right place in the right target cells or tissue [31]. The idea to use a virus as a transporter to insert the functional gene into these cells came after successfully using this procedure in simple goals such as transferring genetic material to a target cell [31]. The viral gene delivery can be carried out via two paths of administration: intravitreal injection, or sub-retinal injection [9]. The transfer of genes into the retina provides many benefits over other tissues: the eye is tiny and closed, needing tiny vector doses to efficiently treat the target, thus reducing exposure to other tissues. [5]

The gene delivery to a particular recipient cell can be constrained at one of several steps shown in Figure 7 [29]. The vector particle must first be attached to a cell receptor and then internalised by interactions with a co-receptor [29]. Vector neutralisation can inhibit or restrict the transduction of the target cells [32]. Innate immune response contributes to the overall immunogenicity of vectors [32]. The vector part must escape from the endosome after viral entry to avoid degradation in its way from the cytoplasm to the nucleus [29]. The virus has to discharge its genome after or during entry into the nucleus by uncoating [29]. DNA with a single strand must be converted into a double-stranded DNA for transgenic expression and stability [29]. The vector DNA combination of the affected cell genome can be genotoxic to the host cell [32]. All these transduction steps can significantly limit gene transfer depending on the serotype and target cell type [29]. 


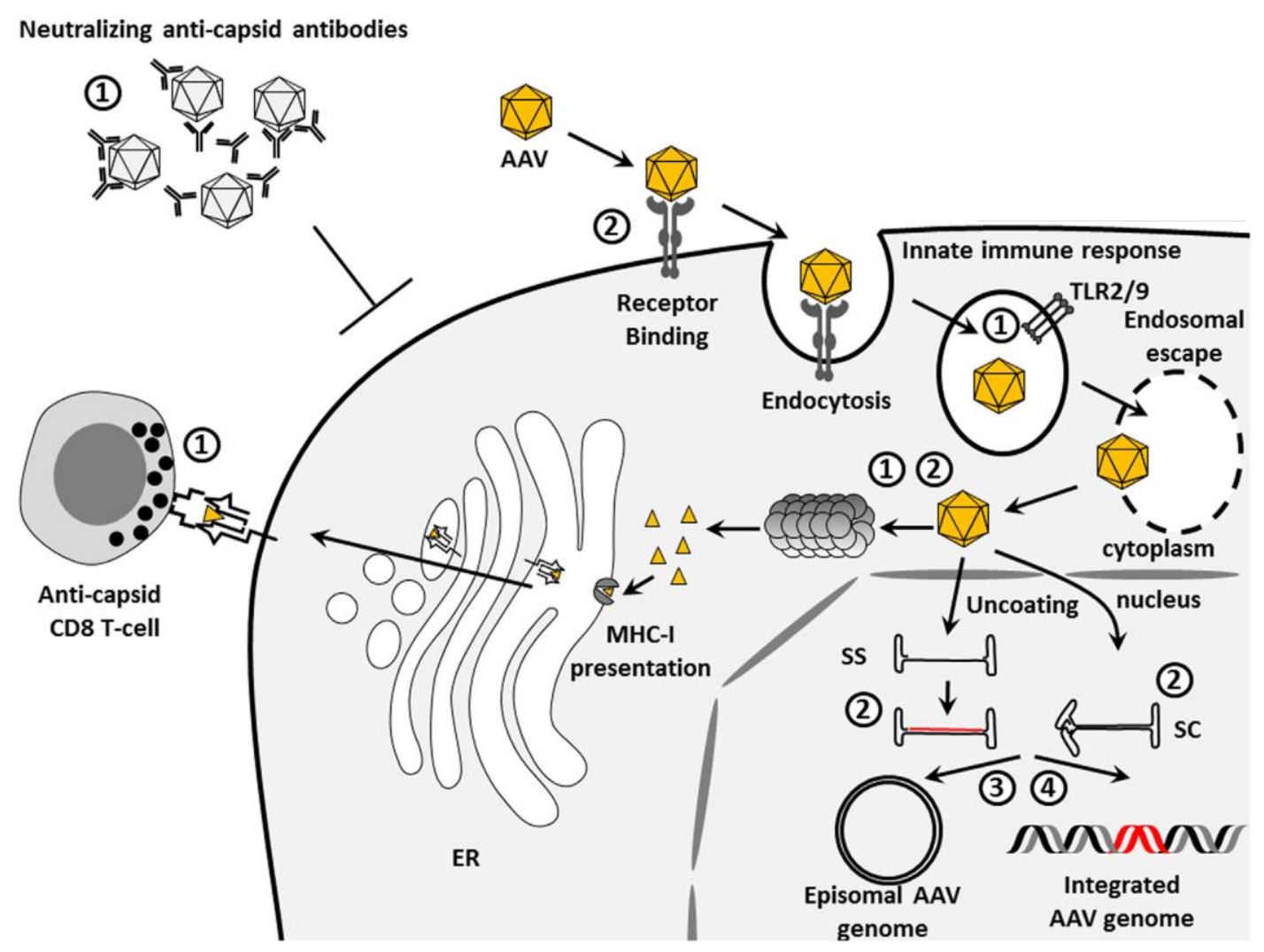

Figure 7. viral gene transfer to a host cell:

This figure which is adopted from Colella at al. illustrates various steps involved in transferring the intended gen loaded on a viral vector (AAV in this example) to be incorporated in the host cell genome [32].

Choosing the right vector is very important to have an overall successful treatment. There are general rules that apply to any successful viral vector which includes: nonreplicating, highly infective, absence of toxicity, flexibility to genetic manipulation, and quickly and reliably produced in the laboratory[31]. Most vectors need to be bioengineered in order to be suitable to be used as a gene therapy vector [31]. On the other hand, ABCA4 gene has its own characteristics that need to be taken into account. The large size ABCA4 coding sequence 6.8 kilobases (kb) and exclusively the expression of ABCA4 in photoreceptor cells [23]. These two qualities necessitate a large cargo capacity vector which efficiently can transduce PRs [23]. 
Most commonly used viral vectors are[31]:

- Adenovirus (Ad)

- Recombinant Adeno-Associated Virus (rAAV)

- Lentivirus (LV)

Each one will be discussed in the following pages.

\subsubsection{Adenovirus:}

Adenovirus (Ad) is a double-stranded DNA virus that is non-enveloped with a capability to load up to $35 \mathrm{~kb}$ of genetic material and to transport it efficiently. It can be developed to large quantities by standard laboratory practices, and it can very effectively infect dividing and non-dividing cells [31].

Ads have a significant drawback in terms of toxicity. Ad is pretty immunogenic, and a host immune reaction can trigger adverse effects, including a decline in transduction ability [31]. Furthermore, the overall population has substantial pre-existing immunity. This not only reduces the serotypes of Ads, which could be used in clinical studies but also prohibit consecutive administration attempts [31].

Current efforts are being made to re-design Ad vectors by lowering their immunogenicity and toxicity, making them safer and more potent. These vectors were thus used in several hundred clinical studies for selective targeting and destruction of tumour cells or for vaccination against pandemic diseases [31]. 


\subsubsection{Lentivirus:}

Lentivirus (LV) is a retroviral genus that includes RNA genome reversely transcribed after infection. LV DNA integrates into the host genome, which makes it the ideal vehicle for stable gene expression following the reverse transcription. Recombinant LV can have a packaging capacity of about $8 \mathrm{~kb}$ of genetic material, has comparatively reduced immunogenicity, and it can infect dividing and non-dividing cells. It is an enveloped virus and can be readily controlled by packaging the virus with heterologous envelope proteins from distinct viral relatives. It is very readily generated in the laboratory for large-and small-scale apps. [31].

The low efficiency of photoreceptors transduction reported in animal models is a limiting factor. This is overcome by a more efficient constitutive CMV promoter [23]. LV may be concerned about the risk of insertion mutations. This may contribute to the disturbance of the host's vital genomic content, which raises ethical and safety concerns [23]. However, later types of LV (non-integrating lentiviral viruses) do not have the enzyme integrase, leading in a virus that can retain extra-genomic vector DNA in linear or circular form without disrupting the target genome.[31]

Using LV for ABCA4 gene delivery has several benefits. Principally, lentiviruses can stably and continuously deliver genes to in vitro transduced cells genome. Second, nondividing cells can be transduced, a vital necessity for terminally differentiated cells such as PR. Thirdly, they can be expressed in relatively large cassette form, which is crucial here, as human ABCA4 complementary cDNAs are nearly $7 \mathrm{~kb}$, which exceed the capacities of the AAV vectors commonly used [23].

StarGen (Oxford Biomedica, Sanofi, SAR422459) is an ABCA4 gene loaded on LV vector designed to regain normal cell photoreceptor function by sub-retinal rout [9]. The initiation of a StarGen Phase I / II clinical study (ClinicalTrials.gov Identifier: NCT01367444) (EudraCT Number: 2010-023111-34) in Stargardt patients has been driven by positive outcomes from animal studies. The clinical trial that started in June 2011 is underway, and it is predicted that 41 patients will be registered and the research should be completed by June 2019. Another phase I / II clinical trial, begun in December 2012, seeks to assess, 15 -year time span, the longterm safety, tolerability and biological activities of StarGen in Stargardt patients (ClinicalTrials.gov Identifier: NCT01736592) [9]. 


\subsubsection{Recombinant Adeno-Associated Virus (rAAV),}

Originally manufactured in the early 1980s, recombinant variants of AAV have since been researched and used more than other choices for viral vectors[31]. The Adeno-associated virus (AAVs), small, icosahedral, single-stranded DNA, has an excellent safety and efficiency profile. To date, there have been successful outcomes of the application of rAAV to Neurodegenerative Diseases in individuals with Parkinson's disease and Leber's inherited Optic Neuropathy (LHON) as demonstrated by safety and efficacy studies [5, 33].

AAV is a non-enveloped virus that contains an estimated 4.7 kilobase $(\mathrm{kb})$ single-stranded DNA genome packaged into a tiny protein shell $[5,29,31]$. rAAV has no immunogenic or toxic reaction after administration at high doses [31]. The presence of more than 100 distinct capsids obtained from the same new AAV serotypes makes it possible to exchange capsids between various serotypes and generate versions of recombinant AAVs with the same genome [5]. RAAV's tropism is very flexible, and it may infect dividing and not dividing cells [31]. The production of high-titer rAAV in tiny and larger scales has been optimised so that the vector is exceptionally acceptable for practically every implementation, from laboratory studies to tiny microbes to pre-clinical or clinical trials [31].

Recombinant AAV drawbacks include small carrying capability. However, this issue has been overcome by different strategies using split genomes method packaging of oversized genomes to overcome AAV cargo limitations. The latest study indicates that specific rAAV serotypes can package and protect the genomes of up to $6 \mathrm{~kb}$ [5]. Also, rAAV stays episomal following infection. This characteristic makes rAAV vulnerable to genetic dilution within rapidly dividing cells and a subsequent loss of transgenic expression [31]. Attention must be drawn to repeat AAV administration when the test requires that this can lead to an immune reaction to the capsid, possibly decrease the effectiveness of the transduction or even cause toxicity in the environment [31].

The chance of effectively packaging large genomes in AAV capsids coupled with rAAV capacity to successfully transduce the affected tissues would allow the emergence of rAAVbased gene therapies for these otherwise untreatable disorders. The transduction of the rAAV2 / 5 in vitro and in vivo mediated results in manifestation and significant morphological and functional improvement of the ocular phenotype in the ABCA4 knockout mouse [5]. 
AAV-2 was found to have significantly less chance of causing innate and adaptive immune responses to its viral capsid or transgenic product [29]. The ability to package up to $8.9 \mathrm{~kb}$ of single-bearer DNA was demonstrated by RAAV vectors with AAV serotype five capsids [5].

\subsubsection{Non-viral delivery systems:}

Non-viral gene delivery systems are undergoing early development at the moment, but it is less popular than viral-based delivery systems. There are many ways to deliver the desired genomic material to the target cell without having to load on a virus. These include Injection of naked DNA between the PR and RPE, Chemical methods which include liposomes, polymers and compacted nano-particles, and physical methods which consist of electroporation, ultrasound, and iontophoresis [23]. Each of these works differently and has its own advantages and disadvantages. The main benefits of non-viral technologies compared to viral technologies are no vector toxicity, no vector immune response, a tremendous transgenic capability and easy and affordable clinical manufacturing [23].

Several trials have shown that viral vectors are more efficient than non-viral gene delivery [23]. Specifically, it is incredibly inefficient to inject "naked" DNA between PR and RPE[23]. "Naked" DNA supplied by non-viral vectors are required to resolve various obstacles, such as extracellular and immune-mediated degradation, and sensor-mediated DNA molecules like toll-like receptors [23]. Another difficulty in photoreceptors is cytoplasmic degradation and the transition through the nuclear envelope [23]. Furthermore, there are additional limits to cellular access to the existence of a physical barrier to the eyes, such as vitreous internal and external limiting membranes, the inter photoreceptors matrix and elevated glycosaminoglycan levels throughout the eye that segregate the DNA [23].

Methods like electroporation and iontophoresis work by electrically stimulating the membrane temporarily to make it more permeable and allow DNA to pass through the cell membranes [23].

DNA conjugations with synthetics or natural cationic compounds, which protect DNA from nuclease-mediated degradation are based on chemical techniques such as liposomes, polymers and compacted nanoparticles [23]. It enables the DND sequence to be transferred to cell membranes through endocytosis and receptor-mediated absorption [23]. The specific CK30NP compacted DNA nanoparticles (NP) showed a significant improvement in the effectiveness of ocular gene transfer and lower levels of immune responsiveness compared with viral 
methods $[9,23]$. Such particles have no theoretical limitations of DNA packaging and have been successfully tested up to $20 \mathrm{~kb}$ [23]. Subsequently, CK30-NPs are administered in the lack of detectable immune reactions or toxicity, resulting in widespread photoreceptor cell transduction [23].

Furthermore, it demonstrates a favourable safety and efficacy profile in human clinical studies for cystic fibrosis following the achievement of CK30-NPs in mediated phenotypic rescue in animal models of the retinitis pigmentosa [23]. The Abca4 knockout mice model of the Stargardt disease was lately studied for delivery of the ABCA4 gene. This study reveals detectable concentrations of ABCA 4 transgenic expression for up to 8 months after the subretinal injection of CK30-NPs containing the human ABCA4 gene [23]. This study demonstrates better restoration of dark adaptation and decreased the accumulation of lipofuscin levels [23]. Continuous development is required of the therapeutic potential of non-viral gene therapy for the retina [9]. 


\subsection{Stem Cells Therapy:}

Degeneration of RPE causes photoreceptor loss in many sight-threatening diseases, such as Stargardt's macular dystrophy [34]. Therefore, stem-cell therapy has significant capacity for treating Stargardt disease by regenerating RPE and preventing vision loss. There are many ways to obtain stem cells includes adult stem cells, human embryonic stem cells (hESCs) and pluripotent stem cells (iPSCs) [9]. Eye and other immune-privileged tissues are likely to be the first stem-cell-based therapies targets.[34].

The RPE cells are critical to maintaining the health and integrity of external retinal, choriocapillaris and photoreceptor cells by serving many functions [34-36].

Providing a new layer of RPE cells can theoretically solve the issue by providing anatomical and physiological support to PR. Despite achievement in many animal models, there are certain constraints, including the likelihood of teratoma transformation and histoincompatibility challenges, in addition to ethical and regulatory issues [34, 35].

This section will discuss different stem cell therapy options to treat ABCA4 retinopathy. 


\subsection{1 human embryonic stem cells:}

Human embryonic stem cells (hESCs) have been regarded, since they were discovered in 1998 , as a possibly useful regenerative medicine instrument $[34,35]$. Recent advancement has been achieved in the production of human ESCs to replace the RPE [9]. Previous attempts have been made to transplant intact plates and suspensions of primary RPE cells in humans with blended outcomes; both in terms of graft survival and sight recovery. [34].

There is proof in macular degeneration models in mice and rats that hESC-derived RPE transplantation can rescue photoreceptors and stop vision loss [34]. Using hESCs as a source of substitute tissue for clinical studies, however, has its own advantages. Besides generating an infinite amount of healthy young cells with presumably decreased immunogenicity, the in-vitro differentiation phase can be regulated to guarantee optimum safety, identity, purity and potency before transplantation into the targeted patient population [9, 34]. This can be further improved by culturing RPE monolayers pre-engineered on artificial Bruch's membrane [36].

HESC derivatives must be free of pathogens, accurately retain its differentiated cell qualities, be highly pure, and free of undifferentiated cells [34]. In animal products, the absence of teratomas, hyperproliferation, cell migration into other organs, the formation of ectopic tissues, immune rejection and adverse consequences must also be extensively tested. [34]. Adverse events linked to the surgical procedure or immunosuppressive regimen should also be explored [9]. Follow-up and additional studies are required to determine the long-term safety and effectiveness of hESC-derived cells as a prospective source of substitution to treat retinal degenerative disease [35].

One hESC-derived clinical trial of RPE cell transplantation was done on patients with AMD and STGD1 for safety and efficacy [35]. No signs of the anterior chamber or vitreous cell or flare is seen post-operatively [35]. There was no structural or functional improvement after the surgery [35]. No evidence of evident immune rejection was detected in patients who underwent transplantation and were able to maintain systemic immunosuppression [35].

Following successful studies in pre-clinical areas, a phase I/II, open-label, nonrandomized, sequential, multi-centred trial to evaluate the safety and tolerance of hESC-RPE transplantation in patients with Stargardt's disease was developed (ClinicalTrials.gov Identifiers: NCT01469832) [9]. While the phase I / II clinical studies have provided positive results, the conclusions must be reviewed carefully, based on the limited participants, short follow-up time and lack of control [9]. 
A study of 13 participants carried out in the United States in 2015 is registered with ClinicalTrials.gov NCT01345006 [34]. After the surgery, immunosuppression was used for 12 weeks [34]. During the follow-up course, no recognisable teratoma formation, hyperproliferation, intraocular inflammation or abnormal growth was observed [34]. The Stargardt macular dystrophy study showed definite functional visual improvement corresponds to the transplant region of the macula [34].

A long-term safety and tolerability assessment of hESC-RPE transplantation in Stargardt patients have been continuous with an expected end date of December 2019. (ClinicalTrials.gov Identifier: NCT02445612) [9]. The goal of this research is to evaluate five years of therapy safety and tolerance after surgery. Future multicenter randomised trials will further assess the potential for the improvement of vision of patients suffering from Stargardt disease or other degenerating retinal dystrophies with hESCs derived transplantation RPE.[9] 


\subsubsection{Bone Marrow-Derived Stem Cells:}

This is an example of adult stem cells where the patient is injected with autologous bone marrow-derived stem cells. These cells are taken from the patient himself so, limited immunogenicity concerns are there. These cells may undergo some genetic modulation using CRISPR/CAS technology to offer come to the definitive genetic sequence [30]. After that, these cells are introduced to the eye by various surgical options, including retrobulbar, subtenon, intravitreal, intraocular, subretinal and intravenous injections [37].

Stem Cell Ophthalmology Treatment Study (ClinicalTrials.gov Identifier: NCT01920867), has 300 participants where they will be followed for 12 months with serial imaging and diagnostic testing with a completion date in August 2019 [37]. In Stem Cell Ophthalmology Treatment Study II (ClinicalTrials.gov Identifier: NCT03011541), Patients will be followed for the same period, but they recruited larger population (500) participants in this trial, and it is proposed to be completed in 2021 [37]. a prospective, nonrandomized, pilot study (ClinicalTrials.gov Identifier: NCT03772938) which aims to analyse the safety and efficacy of intravitreal bone marrow stem cells. At the end of the study, they will assess the safety and efficacy of the treatment. This trial has a much smaller population (30 participants), and it should be completed in 2020.[37] 


\subsection{Pharmacotherapy:}

The pharmacological strategies for treating ABCA4 retinopathy are slowly progressing because it is not a straightforward one. There are many pharmacological agents suggested to regain sight or stop the development of the disease. Most of these agents did not go beyond pre-clinical studies. In order to achieve the desired synergistic pharmacological effect, instead of using high and possible toxic drug doses in order to affect the desired response, two or more medications at each of their individual sub-therapeutic safe dose could be used. [10]. This method has lately been validated by a team to demonstrate protection against light-induced lipofuscin and bisretinoid deposition in the mouse model [10].

ALK-001, fenretinide and A1120 are among the most widely investigated substances for the treatment of Stargardt disease and ABCA4 retinopathy in particular [9]. These three and others will be discussed in term of the mechanism of action, side effects and its constraints.

\subsubsection{Emixustat:}

Emixustat is a new non-retinoid visual process modulator, orally-administered, of retinylamine that inhibits RES (encoded with RPE65 genes), decreasing the transformation of all-trans-retinyl-ester to 11-cis-retinol (Figure 8) and prevents the accumulation of A2E as mentioned in step 5 of section $0[22,38]$. Developed initially to slow geographic atrophy progression in AMD, the treatment was also assessed as a possible treatment for STGD1 [22]. In May 2016, Acucela announced the outcomes of its Phase 2b/3 clinical study, which failed to satisfy its primary endpoint, as there was no statistically significant distinction in the rate of lesion progression or mean a change of BCVA from baseline to month 24 between therapy group relative to placebo [22]. 


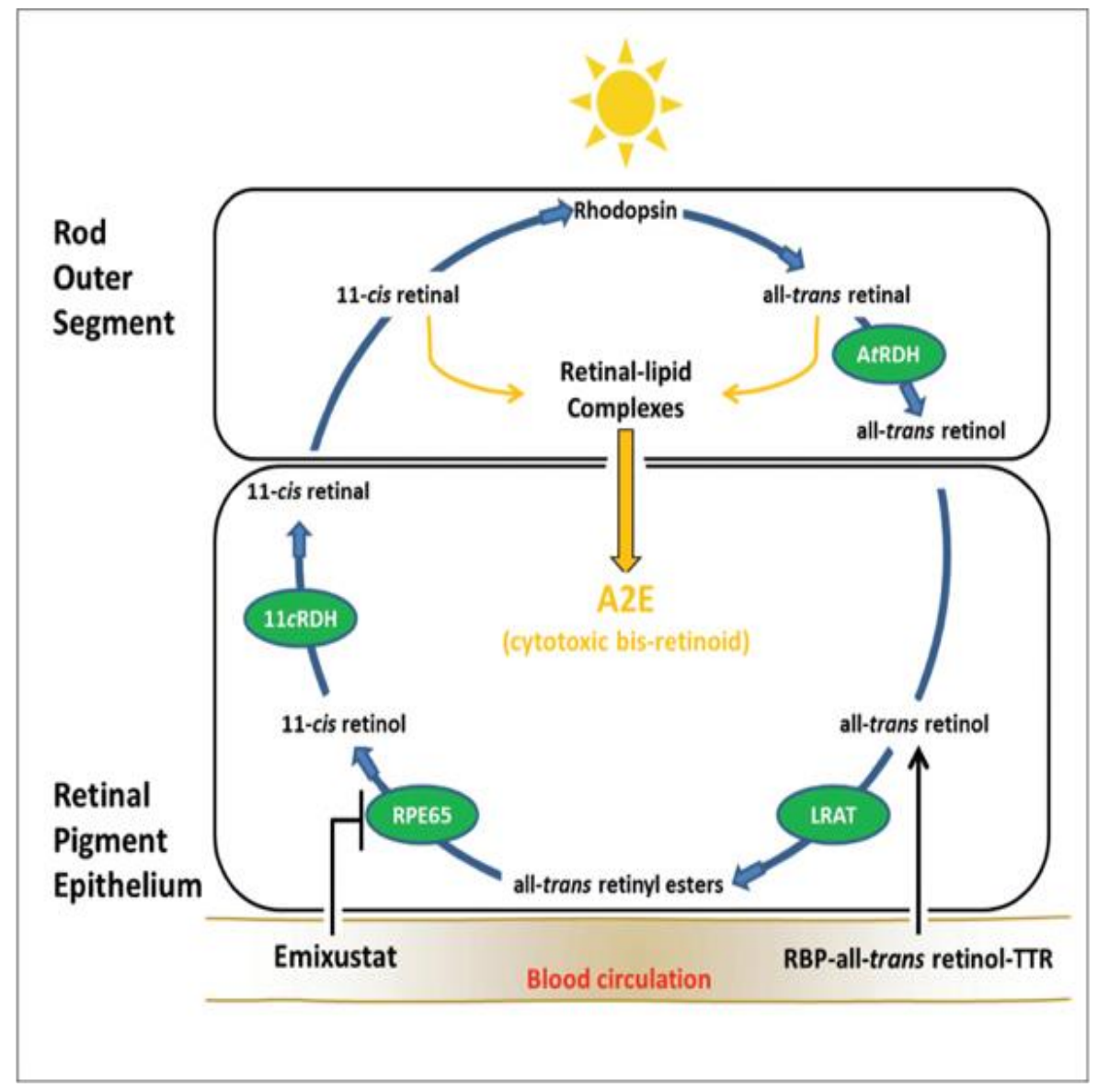

Figure 8. Emixustat site of action.

This figure is adopted from the Lancet publication, which illustrates the site of action of Emixustat about the retinoid cycle [39].

Research on absorption, distribution, and metabolism study was performed with a single dose of emixustat in healthy human participants [38]. Clinical safety and tolerability researches have shown that oral doses of emixustat are correlated with limited adverse reaction [38]. An issue connected with RPE65 inhibition arises from the corresponding reduction of visual responses and temporary decline of light sensitivity resulting from the reduction of the visual chromophore. [10]. Therefore, new treatments that do not directly target visual cycle inhibition may be necessary to prevent an unwanted, even though reversible, side-effect. [10]. 
There is ongoing phase 3 study (ClinicalTrials.gov Identifier: NCT03772665) (EudraCT Number: 2018-003498-82) which is done to determines whether emixustat hydrochloride decreases the rate of progression of macular atrophy relative to placebo in patients with Stargardt disease. In this study, 162 participants all of them with two mutations of the ABCA4 gene or one mutation with a typical STGD appearance of the retina. This study expected to be completed in March 2022.

\subsubsection{Zimura:}

Avacincaptad pegol (Zimura ${ }^{\circledR}$ ) is an inhibitor of the C5 complement system, which impairs C5a signalling and membrane attack complex MAC. It is supplied by intravitreal injection and is being investigated as a possible prospective treatment for a variety of eye diseases, including AMD and Stargardt disease. A phase 1 clinical trial shows that intravitreal Zimura is well-tolerated, and there was some initial effect for AMD patients with a relative dose-dependent progression reduction following monthly injections. By November 2019, a Phase 2 (ClinicalTrials.gov Identifier: NCT03364153) (EudraCT Number: 2017-004783-35) trial should be finalised. [28]

There is one inactive trial at clinicaltrials.gov records which compare Zimura to Sham in Patients With autosomal recessive Stargardt Disease (ClinicalTrials.gov Identifier: NCT03364153) which was started in January 2018 and to should end by September 2020. This study should include 95 participants. In this study, they are using SD-OCT to assess the ellipsoid zone defect progression rate. This study inclusion criteria includes at least two confirmed mutations at the ABCA4 gene. Such studies are of great benefit as it shows patients and clinician if the intervention is worth trying. There are combination treatments also under assessment. Zimura in Combination with LUCENTIS in Patients with Neovascular AMD is completed. ZIMURA is used in Combination with Eylea for IPCV, but it was Withdrawn. Zimura also studied on Subjects with Geographic Atrophy in AMD.

\subsubsection{Docosahexaenoic acid:}

Omega 3 oil is generally believed to be good for our wellbeing. The three-primary omega3 fatty acids are eicosapentaenoic acid (EPA), alpha-linolenic acid (ALA), and Docosahexaenoic acid (DHA) [40]. Fish and other marine products are rich in DHA [40]. Data 
show that macular degeneration incidences are inversely proportional to DHA consumption and that DHA can be protective.[40]

The is a single survey recorded on the Clinical Trials website (NCT00060749) that studies the impact of DHA on ABCA4 retinopathy [40]. DHA is used in this research as a nutritional supplement to explore if macular function in ABCA4 and Stargardt-like macular dystrophy (ELOVL4) individuals can be enhanced [40]. This study was a double-masked, randomised, placebo-controlled, crossover study. In this study, no improvements in mfERG peak amplitude were noted between placebo and DHA supplementation cycles [40]. Visual acuity, visual field and colour confusion also contribute to the conclusion that macular function was not altered in this patient cohort [40]. This study has some of the limitations, such as small sample size [40]. Another one is a carry-over effect in this crossover design [40]. Future studies should be conducted with a more extended period of observation, more population size and without a crossover design and reconsidering ERG as a primary outcome of the study [40]. A comparable survey of 20 individuals with Stargardt's disease, where a smaller dose of DHA used over six months had the same findings. [40].

\subsubsection{ALK-001:}

ALK-001 is a chemically altered vitamin A that resists dimerisation and eventually lipofuscin accumulation, by replacing three hydrogen atoms with deuterium (heavy hydrogen) $[3,10,41]$. As an oral drug, ALK-001 (C20-D3-vitamin A) has been developed, which slows down the dimerisation rate of vitamin $\mathrm{A}$ and prevents the subsequent formation of cytotoxic $\mathrm{A} 2 \mathrm{E}$ and lipofuscinogenesis [3,9]. This substitute reduces the abnormal dimerisation of vitamin $\mathrm{A}$, which is expected to lead to retinal degeneration but without the photoreceptor depriving vitamin A or altering the ocular retinaldehyde concentration. The ALK-001 is, therefore, not a modulator for a visual cycle [10]. Moreover, a shortfall of this type of therapy is that lipofuscin is not removed, but its formation is slowed down [41].

ALK-001 resisted dimerisation of vitamin A and development of A2E and lipofuscin in murine models of Stargardt disease without having any negative implications on the function of the retina [9]. When administered C20-D3-vitamin A to ABCA4 knockout mouse, accumulation levels were four times slower opposed to accumulation levels in the same animal given vitamin $\mathrm{A}$ as shown in Figure 9. As expected, C20-D3-vitamin A administered to Abca4-/- mice for nine months had undistinguishable dark-adapted a- and b-waves, ERG, 
and $20-\mathrm{Hz}$ flicker responses indicating that no adverse effects in electrophysiological function resulted from long-term inhibitors of dimerisation with C20-D3-vitamin A. [42]. These findings further show that administration of C20-D3-vitamin A may be a suitable therapeutic approach to stop the progression of the retinal disease [27]. No side effects to the mice were observed after given the drug for 12 months. [27].

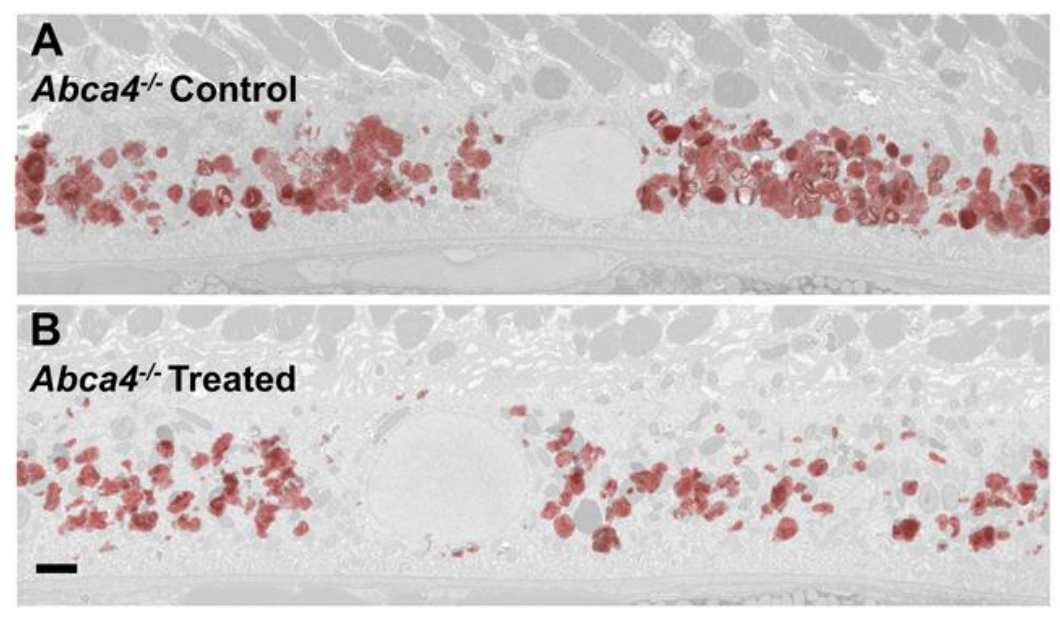

Figure 9.ALK-001 supplementation effect.

This figure is adopted from Charbel et al. [42]. It shows that knockout mice administered either vitamin A (control, A) or C20-D3-vitamin A mice (treated, B). Lipofuscin granules (red) are less copious in RPE (light grey) in the treated mouse.

The successful outcomes of the earlier described pre-clinical studies have led to starting human clinical studies on STGD1 for the oral once-daily C20-D3-vitamin A molecule ALK001 [27]. In 2015, a Phase 1 study (NCT02230228) was concluded with satisfying outcomes to evaluate safety and pharmacokinetics for 40 healthy participants [9]. Alkeus Pharmaceuticals is conducting a Phase 2 clinical trial at the moment on 50 participants, which is expected to complete by March 2022, evaluating ALK-001's efficacy and long-term safety and tolerability in Stargardt disease progression (ClinicalTrials.gov Identifier: NCT02402660)[3, 9, 27, 41].

We would not expect C20-D3-vitamin A to inhibit or increase the toxicity of vision at widely recognisable safe doses than those made by regular vitamin A [27]. No reports of side impacts related to vision, such as delayed dark adaptation or Nyctalopia were reported [10]. 
C20-D3-vitamin A can be used to research the relation between dimerisation, lipofuscin and vision, and as a potential therapeutic option for macular degenerations [27].

\subsubsection{VM200:}

VM200 is an oral molecule presently being preclinically tested and act by reducing ABCA4 dysfunction by toxic byproducts all-trans-retinal [22, 43]. This aldehyde trap reacts with the all-trans-retinal aldehyde group to form a stable Schiff base, so it cannot form A2E $[22,43]$. In the ABCA4 knockout animal model, VM200 was found to maintain the retinal structures which were shown using spectral-domain OCT [22, 43]. Additionally, it preserves retinal functionality, which was confirmed by a high concentration of 11-cis-retinal than controls $[22,43]$. There were no significant toxicities associated with VM200 noticed in this study $[22,43]$. Preclinical trials are ongoing for ABCA4 retinopathy and another therapeutic potential in other diseases [22, 43].

\subsubsection{Isotretinoin}

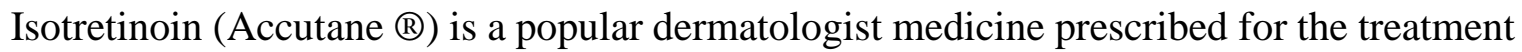
of acne. [20]. Study of its effect on vision and cycle shows that 11-cis-retinol dehydrogenase has an inhibitory effect, as demonstrated in stage 5 of the visual cycle [20,22]. Light activation of rhodopsin results in the formation of all-trans-retinal, the first reactant of toxic $\mathrm{A} 2 \mathrm{E}$ biosynthesis. [22]. Isotretinoin (13-cis-retinoic acid) impacts the last catalytic stage in the visual cycle leading in slow 11-cis-retinaldehyde synthesis and rhodopsin regeneration [20]. Slowing the synthesis of 11-cis-retinal by pharmacologically inhibiting the visual cycle is a practical strategy for restricting the light-dependent synthesis of all-trans-retinal [20]. Isotretinoin also attaches to RPE65 and could thus also block isomerase. [20, 22]. While photoreceptor degeneration is not induced, isotretinoin protects against light damage [22].

Wild-type animals introduced with isotretinoin for two months had a $40 \%$ decrease in the generation of A2E in the RPE opposed to controls. [22]. Sadly, isotretinoin impact can only 
exist at doses where it causes unacceptable adverse effects [20]. Therefore, the therapy of macular degeneration patients in long-term conditions is not appropriate. [20]

\subsubsection{Fenretinide}

A synthetic oral derivative called Fenretinide has been formulated by a Sirion therapeutics [22]. Fenretinide (4-hydroxyphenyl retinamide) or briefly 4-HPRN is a synthetic derivative of vitamin A competing against retinol for the binding of a retinal binding protein in the eye, which prevents the transportation of retinol to RPE [9, 22]. Eliminating the RBP fenretinide complex from the urine will lead to reduced circulatory RBP concentrations and thus reduced levels of vitamin $\mathrm{A}$ in the eye and decelerated A2E biosynthesis $[9,22]$.

In a Stargardt mouse model, preclinical research showed fenretinide drops the concentrations in a dose-dependent circulating RBP-retinol and massively drop buildup levels of A2E.[9, 22]. Mainly, trials have raised issues about the long-term safety profile of fenretinide, as it appears to be a vigorous inducer of apoptosis in many cell kinds, including RPE, the development of hemangiosarcoma in mice and potentially teratogenic features which is problematic for Stargardt disease patients but not AMD patients.[9, 22]. Existing information, therefore, encourage further exploration on both the therapeutic and the safety properties of fenretinide for Stargardt disease.[9]

\subsubsection{A1120}

Because fenretinide safety profile in people with atrophic AMD and Stargardt disease may be inconsistent with long term dosing, identifying innovative structural forms of RBP4 antagonists is remarkably essential [44]. A1120 is a non-retinoid RBP4 antagonist (Figure 10) which is initially designed as a prospective diabetes treatment option [9, 22, 44]. Even though A1120 (ICR-14967) has failed as an effective diabetes medication, it is now being evaluated as a prospective therapy for Stargardt disease and dry AMD [9, 22]. 


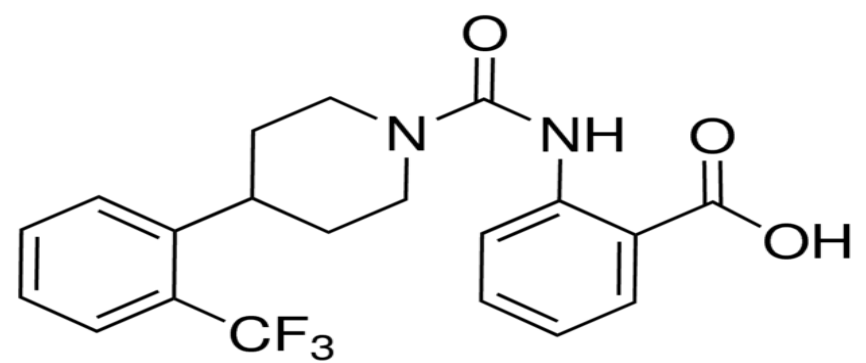

Figure 10. A1120 structure.

Adopted from Merck KGaA [45]. Illustrate the chemical structure of the A1120 molecule.

A preclinical study on a mouse model of Stargardt disease confirms that A1120 can compete with vitamin A for RBP binding and to decrease serum RBP4 levels in a similar to the pathway of fenretinide pathway [9]. In comparison with the control group, ABCA4 knockout mice that have been given A11 20 for six weeks have shown 75\% decreased of serum RBP4 and a 50\% reduction for lipofuscin bisretinoid which in turn decrease A2E accumulation $[9,22]$. On ERG testing, it shows that the ability of A1120 to reduce lipofuscin bisretinoids in the retina may not be associated with significant visual cycle suppression. [22, 44].

Fenretinide and A1120 both share being an antagonist to RBP4. However, A1120 does differ because it is not a retinoid, and it is not an agonist to Retinoic Acid Receptor-alpha (RARa) $[22,44]$. This can spare patients from retinoid-related side effects, including nyctalopia and delayed dark adaptation. [22, 44]. Based on this preclinical information, clinical trials are being developed through cooperative attempts. [22].

\subsubsection{4-Methylpyrazole}

According to ClinicalTrials.gov, 4-Methylpyrazole (4-MP) known as fomepizole and registered under the trademark of Antizol is FDA licensed for human use to treat people with methanol or ethylene glycol toxicity by slowing down the body's capacity to process alcohol. A 4-MP Phase 1 pilot study was carried out in 2006, involving ten subjects [37], in order to treat Stargardt macular dystrophy (ClinicalTrials.gov Identifier: NCT00346853). This research aimed to explore whether taking fomepizole inhibits dark adaptation of the eye. Those with 
Stargardt disease could have a higher likelihood of preserving their residual sight by slowing down these pathways. It has been shown to slow down dark adaptation in animals, with no short-term or long-term side effects, as long as people refrain from any alcohol while the medication is in the body. A single dose of 4-MP stays in your body for approximately 12 hours and can thus prevent dark adaptation for up to 12 hours. Studying 4-MP impacts may guide to successful medical therapy to save the sight of Stargardt patients and also have similar impacts for other macular diseases.

\subsection{Other treatment options:}

There are less popular treatment options which have been investigated but to a lesser extent. These include Heparin-induced Extracorporeal Lipoprotein Precipitation (HELP) Therapy, Saffron supplement, induced pluripotent stem cells, laser therapy, and artificial vision. Other options will be touched on briefly in the discussion section. 


\subsubsection{Microcurrent Stimulation Therapy:}

In 1873 and 1887, Dor and Standish documented on the advantage of electrical eye stimulation in different diseases [8]. Electrical stimulation efficacy findings were reported in laboratory animals and patients with neurodegenerative eye diseases [8]. This result inspired several teams globally to investigate the therapeutic capacity of eye electrical stimulation in animals and humans [8]. After optic nerve injury or light-induced retinal damage, electrical stimulation has been shown to have beneficial impacts on the survival of ganglion cells and retinal cell populations [8].

Trans-corneal electrical stimulation Therapy for Retinal Disease - A randomised, singleblind pilot study in April 2011 involving 80 subjects was conducted [8]. In total, patients were examined nine times, including a baseline visit accompanied by six successive weekly treatment visits and two follow-up exams [8]. Within six consecutive weeks of 30-minute DTL, stimulation improved only the threshold of perception for electrically evoked phosphenes in one patient in the examined parameters [8]. Under the stimulants and exam circumstances noted no statistically detectable impact could be seen on the one side because of brief research that is potentially too limited relative to years or decades of disease or, because of the ineffectiveness of trans-corneal electrostimulation (TES) in Stargardt disease [8]. A successful Stargardt's disease treatment with this kind of therapy needs ongoing research with a larger population and over a long period of at least one year [8]. Electrostimulation, on the other hand, has a range of potential benefits over other treatments: it is considerably less invasive than other methods currently known like intravitreal injections or gene therapy, and it can be done at home regularly by the patient [8]. 


\subsubsection{Removal of Lipofuscin:}

A strategy was believed to break down lipofuscin fluorophores within lysosomes once they have accumulated to a pathological concentration [10, 41]. Enzyme replacement therapy (ERT) for lysosomal storage diseases provides a reliable clinical approach for managing the lysosomal accumulation of toxic undegradable metabolites, which is not directly relevant in ABCA4 retinopathy [41]. This is due to the lack of a native enzyme that degrades lipofuscin fluorophores in RPE cells [41]. Schloendorn and Sparrow earlier identified in cell-free assays several fungal peroxidases capable of degrading $\mathrm{A} 2 \mathrm{E}$, the most prominent lipofuscin fluorophore [41]. A proof-of-concept study is carried out to use both wild type manganese peroxidase $\mathrm{MnP}$ and recombinant $\mathrm{MnP}$ to break down $\mathrm{A} 2 \mathrm{E}$ and other fluorophores lipofuscin, reduce $\mathrm{A} 2 \mathrm{E}$ levels and toxicity associated with $\mathrm{A} 2 \mathrm{E}$ packed cells, and lower A2E load for ABCA4 mouse model [41]. There was a significant reduction in the treatment group, up to $31 \%$ compared to control in this experiment [41]. Because glycans are also critically important in recognition of the immune system, humanising the enzyme's glycosylation could also help with any immune-related toxicity [41]. However, extra efforts are needed to improve effectiveness and to define and attenuate the causes of frequent dose toxicity [41].

Photodynamic therapy is flowed by intravitreal horseradish peroxidase injection, and blue light exposure, transmission-electron microscope (TEM) pictures of RPE was analysed, suggesting that extraction of lipofuscin from RPE is possible without disturbing the RPE cell layer [10]. Oral Remofuscin (Katairo) was initially developed as an acid pump antagonist (APA) to treat gastroesophageal reflux disease (GERD). Its active ingredient is soraprazan, a small molecule that stimulates lipofuscin exocytosis from RPE cells [10]. In ABCA4 Knockout mouse, lipofuscin granules were reduced by more than 2 -fold following intravitreal injection of Remofuscin [10]. A multi-national, multi-centre, double-masked, placebo-controlled proof of concept trial to evaluate the safety and efficacy of oral soraprazan in Stargardt disease started in April 2019 (EudraCT Number: 2018-001496-20). It is going to be conducted in up to four European countries by the enrolment of approximately 90 Stargardt disease patients.

\subsubsection{IVI:}

STGD disease course is progressive central atrophy and therefore, the loss of central visual capacity [46]. Choroidal neovascularisation (CNV) associated with STGD is uncommon, but 
it may lead to a further deterioration of visual acuity. [47]. It may develop as a natural course of the disease or due to trauma to Bruch's membrane during the surgical procedure associated with previous surgical treatment options. Because the CNV growth is rare, which uncommonly can complicate ABCA4 retinopathy, there is a restrict data availability of its management options [46, 47]. There are many possibilities which are currently widely used as intravitreal injection IVI, including aflibercept (Eylea), ranibizumab (Lucentis), and bevacizumab (Avastin). These medications are used in a different regimen to treat neovascular AMD, Diabetic retinopathy, retinal vascular occlusion and other eye diseases. Lucentis is a recombinant, humanised, monoclonal antibody antigen-binding fragment which neutralises all forms of (VEGF), successfully used in treating neovascular AMD and assessed for treatment of ABCA4 neovascularisation. [46].

A 26-year-old patient with the right sub-foveal CNV offered an option of ranibizumab intravitreal injection because he has a large size lesion [46]. In this patient, intravitreal ranibizumab halted both the $\mathrm{CNV}$ leakage and progression, but there was no significant improvement of BCVA at the end of follow up visits [46].

Another trial included three patients with ABCA4 retinopathy verified by molecular testing who were followed-up monthly over 24 months [47]. They all got baseline ranibizumab injection and underwent a pro re nata PRN retreatment scheme depending on OCT liquid detection or FFA leakage. [47]. The total number of injections was 6 to 8 at 12 months, which increased to 9 to 14 at 24 months period. [47]. CNV did not show any activity in two cases during the 24-month examination, while the third case, show intra-retinal fluid and leakage, which was advised to undergo further treatment. [47]. Also, outer retinal tubulations (ORT) developed over time in all cases, and all eyes demonstrated an overall increase in RPE atrophy [47]. Although this research demonstrates that 24 months of treatment with ranibizumab shows no improvement in visual function, anti-VEGF therapy may exacerbate the development of STGD atrophy [47]. As mentioned previously, inflammatory mechanisms perform an essential part of this disease's pathophysiology. A more extensive strategy to $\mathrm{CNV}$ complicated ABCA4 retinopathy may be needed, incorporating anti-VEGF treatment with other therapeutic options to accomplish a neuroprotective effect and inflammatory control [47]. Further studies should define the best therapy for CNV related to STGD. 


\section{Discussion:}

Due to a high in genotypic and phenotypic variability, numerous synonymous are used to refer to $\mathrm{ABCA} 4$ retinopathy. ABCA4 /ABCR retinopathy, Stargardt disease, fundus flavimaculatus retinal dystrophy and other terms are used to describe the same disease. Furthermore, there is variability in the literature in the $\mathrm{ABC} 4$ transporter gene expression location, as discussed in section 3.2.3. This leading to a question of why peripheral retina is not involved or slightly involved if it is expressed in all photoreceptors. One explanation is due to the increased ratio of photoreceptors to RPE cells [20]. This contradicts what is known about the one to one cone to RPE ration in the macula.

Furthermore, what is the effect of the role of ABCA4 transported in the brain? Are there any neurological abnormalities manifestations in ABCA4 retinopathy? These questions should be investigated, and it can lead to new approaches to deal with such disease [14].

Having an animal model is of great benefit for the disease-related studies in the short and long run. It helped in understanding the various mechanisms and roles of different molecules in the visual cycle. Still, one of the limitations of ABCA4 knockout mice is being not consistent with the human phenotype [48]. Many variations in ABCA4 mutations which are estimated to exceed 900 in some studies are present [2]. Coexistence of tow mutations or more along with environmental modifiers contributes to ABCA4 retinopathy high heterogenicity $[10,24,25$, 49]. This leads to difficulty in assessing and staging the disease, which is crucial in the clinical trials inclusion and exclusion criteria. Clinical trials endpoints are very variable between studies, and they mostly have BCVA as one of them or the only one. This is not applicable in this disease, as discussed in the relevant section 4.

Gene therapy is an attractive and promising field which taking advantage of ABCA4 retinopathy being a recessive disease. Replacing one copy of the gene can restore normal function. This technique is approved for another related disease. After managing the big cargo issue associated with the ABCA4 gene using the recombinant viral delivery system, many trials are in progress to assess the long-term safety and efficacy of these treatment options. On the other hand, non-viral systems have no issue with the size of the gene but, with it integrating these freely flatting DNA segment into a photoreceptor cell genome.

Stem cells therapy for ABCA4 retinopathy work on the principle that photoreceptor cells death is secondary to RPE cell death. This means that there is a window of opportunity where this treatment can restore or at least sustain the vision. This option mostly dominates the 
clinical trials (at least in clinicaltriilas.gov) in ABCA4 retinopathy. Both discussed options deal with RPE damage and replace it surgically, which has its own additional risks. On the other hand, Photoreceptors will continue to accumulate lipofuscin in the new and probably fragile RPE, which will ultimately result in their damage. This surgical procedure may result in Bruch's membrane damage, or it may get damaged throughout the disease process. Substituting RPE cells cultured on artificial Bruch's membrane may be a more sensible approach. Still, consideration like long term effect of artificial Bruch's membrane on the adjacent tissue and the vast difference metabolic demand by photoreceptors and the newly growing and struggling RPC cells in their new environment should be taken into account.

Many pharmacological compounds are being investigated for ABCA4 retinopathy disease, 9 of them being discussed in this paper. This account for more than the sum of all other options combined. This makes sense as these compounds are much less complicated to produce, manipulate, deliver, test and withdraw for the body as compared to other more invasive and permanent options. Especially in the presence of an animal model which make things more justifiable when it reaches to clinical trials stage. Emixustat is a promising one as there is an ongoing phase 3 trial on quite a large population. Zimura is IVI, which smartly uses a popular delivery method to the site of action without taking the risk of systemic absorption and systemic side effects. It can be alternated with other approved IVI drugs to treat CNVs. Still, this approach requires repetitive injections which may be difficult for visually impaired patients and very costly for the government to provide as a free long run treatment for such rare disease. With ALK-001 safety profile and excellent preclinical trials, ongoing clinical trials are promising, and their results are locked forward. A1120 seems to be a more realistic option than fenretinide because its safety in the long term and the side effects associated with fenretinide. Nyctalopia and impaired dark adaptation are known side effects to a specific pharmacological options, which doubt its practicality if used in real life.

There are many other treatment options which are not discussed in this paper due to lack of sufficient literature on them or because there are no registered trials on them at the database registry. Still, this does not mean that they are not applicable or not valid, but it needs to be investigated and tried. These options include but not exclusive to Heparin-induced Extracorporeal Lipoprotein Precipitation (HELP) Therapy, Saffron supplement, induced pluripotent stem cells, laser therapy, and artificial vision. HELP therapy was studied in 22 patients with AMD disease, which completed in 2016 (ClinicalTrials.gov Identifier: NCT01840683) with no report up to date. Saffron supplement proposed to have a 
neuroprotective role against oxidative damage. Keeping in mind that saffron has plenty of antioxidant compounds and it is safe and almost free of side effects (in the acceptable doses), my influence and improve ERG which is the outcome of 2017 clinical trial (ClinicalTrials.gov Identifier: NCT01278277) [37]. Induced pluripotent stem cells are more complicated than embryonic stem cells as it needs to be reprogramed to behave and act like embryonic stem cells, then to again utilise it to form RPE cells or other cell types. This technique is promising as it can utilise any adult cell to produce an infinite number of embryonic stem cells with identical genetic makeup.

On the other hand, this technology facing more technical, ethical, and regulatory difficulties little more than other stem cells therapy options and much more than gene therapy options. Laser therapy is widely used in the treatment of proliferative diabetic retinopathy treatment. It is mostly used to control CNV growth, which is induced by the ischemic area. The same principle applies in CNV complicated ABCA4 retinopathy. In section 5.4.3, we discussed the treatment of CNV by using IVI, which is probably applicable for laser therapy. Low vision aids can help people with a limited vision where other options cannot be used or the patients not welling to undergo these treatment options. These aids can support them to navigate at home and do their main live activities. Artificial vision is reserved for the end stag patients where all other options cannot be utilized any more. This approach is evolving rapidly, and it has its s own challenges. One of the popular projects in this topic is BrainPort, which has FDA approval. Still, this type of gadgets has many limitations but because no other options available for these patients (usually at middle age in ABCA4 retinopathy) it may serve their needs.

There are many challenges which need to be dealt with in order to have a successful potent ABCA4 retinopathy therapy. First, we need to have a deeper understanding of the differences and similarities between different photoreceptor types. Besides that, we need to have a better molecular understanding of the visual cycle steps. As this disease is often a childhood disease, special consideration should be taken into account to the development of the visual cortex in these children. A financial issue arises in the cost-effectiveness to treat patients with the underdeveloped visual cortex. Another issue is that the structural damage proceeds the functional damage. Because of that, it is difficult to identify and screen their people at the right time. Because this disease is rare, recruiting a large number of patients for multiple trials is very difficult.

$\mathrm{ABCC} 7$ gene is a protein kinase A-dependent chloride channel that expressed in exocrine gland[25]. Mutation int this gen result in Cystic Fibrosis CF, which is one of the most common 
fatal childhood conditions, with a defective lung, pancreatic, sweat and intestinal exocrine activities [25]. A lesson from CF is to be learned as it finally has its own FDA treatment. Tezacaftor / ivacaftor is a small molecule stimulating Cystic fibrosis transmembrane conductance regulator CFTR. [50]. This therapy choice provides CFTR correctors and potentiators that adhere to the protein component of the CFTR mutation in order to enhance its processing and functionality [50]. This treatment has excellent safety and reliability profile, and the same principle should be investigated for the ABCA4 gene.

In my opinion, there is a great hope to have at least one treatment options for ABCA4 retinopathy shortly. I hope to see more studies on some sustained-release, not retinoid modulator which has the benefit of being near to the target tissue using minor surgical procedure, last for few months and overcome the systemic absorption and toxicity issue. 


\section{Conclusion:}

ABCA4 retinopathy is a devastating hereditary macular dystrophy that leads to vision loss at a young age. Mutations of the ABCA4 gene lead to an abnormal accumulation of lipofuscin in the retinal epithelium, causing RPE and PR degeneration and ultimately, visual impairment. Although no established efficient therapy for Stargardt patients is currently available, a range of recent research has progressed to create novel therapy techniques, including gene therapy, stem cell therapy, pharmacotherapy, and others. The evolving gene therapy method is designed to substitute the non-functional copy with a functional ABCA4 gene via different methods. StarGen and other projects of lentivirus vector rAAV vector containing the ABCA4 gene, has successfully moved for a preclinical to early clinical stage. These trials are highly expected to result in clinical outcomes, which will revolutionise its field. Non-viral transport techniques, such as the use of nanoparticles and iontophoresis methods, may have certain benefits over viral vectors which encouraged their further improvement. Stem cell treatment uses human embryonic stem or allograft cell regeneration techniques in patients with Stargardt disease to regenerate RPE. Its' trials are promising, but it is facing ethical and regulatory issues which are partially shared with gene therapy technique. Emixustat, Zimura, ALK-001, fenretinide, A1120 and other pharmacologically manufactures drugs proposed to limit excessive retinal lipofuscin buildup in the RPE. These molecules are widely studied and are currently at various clinical trials stages to treat ABCA4 retinopathy specifically. Continued research into new therapeutics options for $\mathrm{ABCA} 4$ retinopathy will encourage the innovation of efficient therapy by a combined effort from the science and medical society and supported by societies and governments. Stopping visual losses in thousands of kids and young people who are annually diagnosed with this disease is at the horizon. 
References:

1. Miraldi Utz, V., et al., Gene therapy for RPE65-related retinal disease. Ophthalmic Genet, 2018. 39(6): p. 671-677.

2. Lambertus, S., et al., Highly sensitive measurements of disease progression in rare disorders: Developing and validating a multimodal model of retinal degeneration in Stargardt disease. PLoS One, 2017. 12(3): p. e0174020.

3. Tanna, P., et al., Stargardt disease: clinical features, molecular genetics, animal models and therapeutic options. Br J Ophthalmol, 2017. 101(1): p. 25-30.

4. Tanna, P., et al., Cross-Sectional and Longitudinal Assessment of the Ellipsoid Zone in Childhood-Onset Stargardt Disease. Transl Vis Sci Technol, 2019. 8(2): p. 1.

5. $\quad$ Allocca, M., et al., Serotype-dependent packaging of large genes in adeno-associated viral vectors results in effective gene delivery in mice. J Clin Invest, 2008. 118(5): p. 1955-64.

6. Fishman, G.A., Historical evolution in the understanding of Stargardt macular dystrophy. Ophthalmic Genet, 2010. 31(4): p. 183-9.

7. Kaplan, J., et al., A gene for Stargardt's disease (fundus flavimaculatus) maps to the short arm of chromosome 1. Nat Genet, 1993. 5(3): p. 308-11.

8. Rock, T., et al., [Effects of transcorneal electrical stimulation in patients with Stargardt's disease]. Ophthalmologe, 2013. 110(1): p. 68-73.

9. Lu, L.J., J. Liu, and R.A. Adelman, Novel therapeutics for Stargardt disease. Graefes Arch Clin Exp Ophthalmol, 2017. 255(6): p. 1057-1062.

10. Sears, A.E., et al., Towards Treatment of Stargardt Disease: Workshop Organized and Sponsored by the Foundation Fighting Blindness. Transl Vis Sci Technol, 2017. 6(5): p. 6.

11. Molday, R.S., ATP-binding cassette transporter ABCA4: molecular properties and role in vision and macular degeneration. J Bioenerg Biomembr, 2007. 39(5-6): p. 507-17.

12. Walia, S. and G.A. Fishman, Natural history of phenotypic changes in Stargardt macular dystrophy. Ophthalmic Genet, 2009. 30(2): p. 63-8.

13. Dysli, C., et al., Fluorescence Lifetime Imaging in Stargardt Disease: Potential Marker for Disease Progression. Invest Ophthalmol Vis Sci, 2016. 57(3): p. 832-41.

14. Tsybovsky, Y., R.S. Molday, and K. Palczewski, The ATP-binding cassette transporter ABCA4: structural and functional properties and role in retinal disease. Adv Exp Med Biol, 2010. 703: p. 105-25.

15. Allikmets, R., et al., A photoreceptor cell-specific ATP-binding transporter gene $(A B C R)$ is mutated in recessive Stargardt macular dystrophy. Nat Genet, 1997. 15(3): p. 236-46.

16. Strauss, R.W., et al., Assessment of estimated retinal atrophy progression in Stargardt macular dystrophy using spectral-domain optical coherence tomography. Br J Ophthalmol, 2016. 100(7): p. 956-962.

17. Chen, L., et al., Multi-platform imaging in ABCA4-Associated Disease. Sci Rep, 2019. 9(1): p. 6436.

18. Fujinami-Yokokawa, Y., et al., Prediction of Causative Genes in Inherited Retinal Disorders from Spectral-Domain Optical Coherence Tomography Utilizing Deep Learning Techniques. J Ophthalmol, 2019. 2019: p. 1691064.

19. Kolb H, F.E., Nelson R., Webvision: The Organization of the Retina and Visual System [Internet]. Salt Lake City (UT): University of Utah Health Sciences Center, 1995.

20. Travis, G.H., et al., Diseases caused by defects in the visual cycle: retinoids as potential therapeutic agents. Annu Rev Pharmacol Toxicol, 2007. 47: p. 469-512.

21. Grossniklaus, H.E., E.E. Geisert, and J.M. Nickerson, Introduction to the Retina. Prog Mol Biol Transl Sci, 2015. 134: p. 383-96.

22. Hussain, R.M., et al., Stargardt macular dystrophy and evolving therapies. Expert Opin Biol Ther, 2018. 18(10): p. 1049-1059.

23. Auricchio, A., I. Trapani, and R. Allikmets, Gene Therapy of ABCA4-Associated Diseases. Cold Spring Harb Perspect Med, 2015. 5(5): p. a017301.

24. Sullivan, J.M., Focus on molecules: ABCA4 (ABCR)--an import-directed photoreceptor retinoid flipase. Exp Eye Res, 2009. 89(5): p. 602-3. 
25. (MD), D.M.B., The Human ATP-Binding Cassette (ABC) Transporter Superfamily [Internet]. National Center for Biotechnology Information (US), 2002

26. Molday, L.L., A.R. Rabin, and R.S. Molday, ABCR expression in foveal cone photoreceptors and its role in Stargardt macular dystrophy. Nat Genet, 2000. 25(3): p. 257-8.

27. Kaufman, Y., L. Ma, and I.J.J.o.B.C. Washington, Deuterium enrichment of vitamin A at the C20 position slows the formation of detrimental vitamin A dimers in wild-type rodents. 2011. 286(10): p. 7958-7965.

28. Kassa, E., et al., Complement inhibition as a therapeutic strategy in retinal disorders. Expert Opin Biol Ther, 2019. 19(4): p. 335-342.

29. Warrington, K.H., Jr. and R.W. Herzog, Treatment of human disease by adeno-associated viral gene transfer. Hum Genet, 2006. 119(6): p. 571-603.

30. Nair, J., et al., Translatable gene therapy for lung cancer using Crispr CAS9-an exploratory review. Cancer Gene Ther, 2019.

31. Sandro, Q., K. Relizani, and R. Benchaouir, AAV Production Using Baculovirus Expression Vector System. Methods Mol Biol, 2019. 1937: p. 91-99.

32. Colella, P., G. Ronzitti, and F. Mingozzi, Emerging Issues in AAV-Mediated In Vivo Gene Therapy. Mol Ther Methods Clin Dev, 2018. 8: p. 87-104.

33. Wan, X., et al., Efficacy and Safety of rAAV2-ND4 Treatment for Leber's Hereditary Optic Neuropathy. Scientific reports, 2016. 6: p. 21587-21587.

34. Schwartz, S.D., et al., Embryonic stem cell trials for macular degeneration: a preliminary report. Lancet, 2012. 379(9817): p. 713-20.

35. Song, W.K., et al., Treatment of macular degeneration using embryonic stem cell-derived retinal pigment epithelium: preliminary results in Asian patients. Stem Cell Reports, 2015. 4(5): p. 860-72.

36. Warnke, P.H., et al., Primordium of an artificial Bruch's membrane made of nanofibers for engineering of retinal pigment epithelium cell monolayers. Acta Biomater, 2013. 9(12): p. 9414-22.

37. ClinicalTrials.gov. 2019.

38. Fitzsimmons, M.E., et al., Disposition, profiling and identification of emixustat and its metabolites in humans. Xenobiotica, 2018. 48(6): p. 592-604.

39. Lancet publication: Phase $1 b$ Data for QLT091001 (Oral 9-cis retinoid treatment) in subjects with LCA. 2019, Rotterdam Ophthalmic Institute.

40. MacDonald, I.M. and P.A. Sieving, Investigation of the effect of dietary docosahexaenoic acid (DHA) supplementation on macular function in subjects with autosomal recessive Stargardt macular dystrophy. Ophthalmic Genet, 2018. 39(4): p. 477-486.

41. Moody, K.J., et al., Recombinant Manganese Peroxidase Reduces A2E Burden in AgeRelated and Stargardt's Macular Degeneration Models. Rejuvenation Res, 2018. 21(6): p. 560-571.

42. Charbel Issa, P., et al., Rescue of the Stargardt phenotype in $\langle e m\rangle$ Abca4</em> knockout mice through inhibition of vitamin A dimerization. 2015. 112(27): p. 8415-8420.

43. Hussain, R.M., et al., Pharmacotherapy of retinal disease with visual cycle modulators. Expert Opin Pharmacother, 2018. 19(5): p. 471-481.

44. Dobri, N., et al., A1120, a nonretinoid RBP4 antagonist, inhibits formation of cytotoxic bisretinoids in the animal model of enhanced retinal lipofuscinogenesis. Invest Ophthalmol Vis Sci, 2013. 54(1): p. 85-95.

45. A1120. 2019, Merck KGaA, Darmstadt, Germany.

46. Querques, G., et al., Intravitreal ranibizumab (Lucentis) for choroidal neovascularization associated with Stargardt's disease. Graefes Arch Clin Exp Ophthalmol, 2008. 246(2): p. 319-21.

47. Battaglia Parodi, M., et al., Ranibizumab for subfoveal choroidal neovascularisation associated with Stargardt disease. Br J Ophthalmol, 2015. 99(9): p. 1268-70.

48. Cideciyan, A.V., et al., Mutations in ABCA4 result in accumulation of lipofuscin before slowing of the retinoid cycle: a reappraisal of the human disease sequence. 2004. 13(5): p. 525-534. 
49. Singh, R., et al., Longitudinal follow-up of siblings with a discordant Stargardt disease phenotype. Acta Ophthalmol, 2014. 92(4): p. e331-2.

50. Walker, S., et al., A phase 3 study of tezacaftor in combination with ivacaftor in children aged 6 through 11 years with cystic fibrosis. 2019. 